\title{
Two-scale homogenization of abstract linear time-dependent PDEs
}

\author{
Stefan Neukamm, Mario Varga and Marcus Waurick
}

\begin{abstract}
Many time-dependent linear partial differential equations of mathematical physics and continuum mechanics can be phrased in the form of an abstract evolutionary system defined on a Hilbert space. In this paper we discuss a general framework for homogenization (periodic and stochastic) of such systems. The method combines a unified Hilbert space approach to evolutionary systems with an operator-theoretic reformulation of the well-established periodic unfolding method in homogenization. Regarding the latter, we introduce a well-structured family of unitary operators on a Hilbert space that allows to describe and analyze differential operators with rapidly oscillating (possibly random) coefficients. We illustrate the approach by establishing periodic and stochastic homogenization results for elliptic partial differential equations, Maxwell's equations, and the wave equation.
\end{abstract}

Keywords: periodic and stochastic homogenization, unfolding, abstract evolutionary equations, Maxwell's equations

\section{Contents}

1 Introduction $\quad 1$

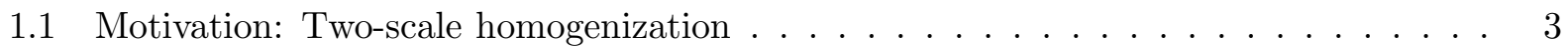

$\begin{array}{lll}2 & \text { The operator-theoretic setting for unfolding } & 7\end{array}$

3 Homogenization of (abstract) elliptic problems $\quad 10$

4 Homogenization of abstract evolutionary equations $\quad 13$

4.1 A Hilbert space framework for evolutionary equations . . . . . . . . . . . . . . 13

4.2 Applications to time-dependent-type problems $\ldots \ldots \ldots \ldots$

5 Examples 19

5.1 Examples of unfolding operators . . . . . . . . . . . . . . . . 19

5.2 Periodic homogenization of elliptic equations . . . . . . . . . . . 25

5.3 Stochastic homogenization of Maxwell's equations . . . . . . . . . . . . . 26

5.4 Stochastic homogenization of some mixed type equations . . . . . . . . . . . 31

\section{Introduction}

In this work we propose an abstract strategy for homogenization of evolutionary equations. The methods we present are based on a combination of three concepts: The abstract unified theory for 
evolutionary equations introduced in [26] (see also [19, 27]), their homogenization theory developed in [40] (with plenty of applications, see references below), and the stochastic unfolding procedure recently introduced in $[23,14]$.

According to [26] (see also [27, 29, 46] and the references therein) a large variety of linear evolutionary equations of mathematical physics can be recast in the following form:

$$
\left(\mathcal{M}\left(\partial_{t, \nu}\right)+A\right) u=F,
$$

where $z \mapsto \mathcal{M}(z)$ denotes a family of linear bounded operators on a Hilbert space $H$ and $A$ is a skewselfadjoint densely defined operator on $H ; \mathcal{M}\left(\partial_{t, \nu}\right)$ is defined in the sense of an explicit functional calculus for $\partial_{t, \nu}$, the time derivative, which is established as a boundedly-invertible operator. We refer to [26] (see also [32, 46, 27, 36, 35]) for existence and other essential results regarding the above equation. We briefly recall the most important ingredients of this framework in Section 4.1.

In many applications one is interested to describe physical properties of systems (e.g., composites, alloys, metamaterials) that feature material heterogeneities on a small length (or time) scale, say $\varepsilon \ll 1$. In the above described framework this may be described by equations of the form

$$
\left(\mathcal{M}_{\varepsilon}\left(\partial_{t, \nu}\right)+A\right) u=F
$$

where $\mathcal{M}_{\varepsilon}$ denotes a sequence of operators with "coefficients oscillating on scale $\varepsilon$ ". The goal of homogenization is to derive a simplified, effective equation, say an equation of the form

$$
\left(\mathcal{M}_{0}\left(\partial_{t, \nu}\right)+A\right) u=F
$$

that captures the large scale properties of the original system. This is typically achieved by studying the asymptotics $\varepsilon \rightarrow 0$, and by proving that the solution of (1) converges in a suitable sense to the solution of the effective problem (2) as $\varepsilon \rightarrow 0$. In the context of abstract evolutionary equations this has been studied in [40, 41, 42, 43, 44, 47, 46, 48, 49]. Apart from [41, 43, 49], the results obtained in these works are typically $H$-compactness statements, that is, it is shown that given a family of operators $\left(\mathcal{M}_{\varepsilon}\right)$ (in a suitable class), it is possible to find (i) a subsequence along which homogenization occurs and (ii) a "homogenized" operator $\mathcal{M}_{0}$ that appears in the homogenized equation and that possibly belongs to a larger class. In applications (e.g., modelling of microstructured materials) the operator $\mathcal{M}_{\varepsilon}$ typically originates from a partial differential equation with coefficients that rapidly oscillate on scale $\varepsilon$ and that encode the specific form of the material's microstructure. In the references $[41,43,49]$ for particular equations, some $H$-convergence results have been obtained (using certain properties of the microstructure providing explicit formulas for $\mathcal{M}_{0}$ ) rather than $H$-compactness, only. Anyhow, it is desirable to incorporate information on the microstructure into the general abstract operator-theoretic approach, and to establish a microstructure-properties relation that allows to uniquely characterize the homogenized operator $\mathcal{M}_{0}$ based on the information on the microstructure.

In the classical field of homogenization of partial differential equations, the microstructureproperties relation often comes in form of homogenization formulas based on correctors and cellproblems. Moreover, various homogenization methods exist that explicitly exploit structural properties of the microstructure. In particular, two-scale convergence methods are convenient for problems involving periodic or random coefficients oscillating on a small (physical) scale. Periodic two-scale convergence $[24,1,18]$ and the method of periodic unfolding $[8,39,9,20]$ are well-suited for problems involving periodic coefficients. For stochastic homogenization problems (e.g., for 
models describing random heterogeneous materials) the notion of stochastic two-scale convergence $[4,2,51,13]$ and the stochastic unfolding method [14,23] are available as convenient tools.

In this paper we join the idea of the stochastic/periodic unfolding procedure with the homogenization approach for abstract evolutionary equations of the form (1) (that is we combine $[25,4,23]$ with the ideas rooted in $[40,41])$. In particular, we introduce an abstract family of operators which present a generalization of the stochastic unfolding operator (see Section 2). Upon assuming a set of structural assumptions for the abstract unfolding operator, we derive a suitable homogenization result for a system of form (1). A merit in this procedure (in contrast to earlier works on abstract evolutionary equations) is that the structural conditions we assume allow us to incorporate information on the microstructure and to obtain an explicit description of the effective model. The abstract homogenization result we obtain covers a large variety of problems. To illustrate this, we specifically reconsider periodic homogenization of elliptic partial differential equations, and obtain (as simple corollaries of our abstract theorem) stochastic homogenization results for the Maxwell's equations and the wave equation. Additionally, we derive corrector type results for the considered examples that are based on specific properties of the partial differential equation under consideration.

Structure of the paper. In order to motivate the theory, in Section 1.1 we recall the standard setting for stochastic homogenization and provide the definition of stochastic unfolding. Section 2 provides the definitions of the unfolding operator and two-scale convergence from an abstract point of view. In the rest of that section we present some important properties of the latter notions. Section 3 is devoted to the derivation of a homogenization result for an abstract elliptic type problem. In Section 4.1 we briefly recall the setting for abstract evolutionary equations. Section 4.2 provides a homogenization result for an abstract evolutionary equation. Section 5 treats some particular examples of the previously discussed abstract theory.

\subsection{Motivation: Two-scale homogenization}

In this section we recall some classical results and concepts from periodic and stochastic homogenization of second order, divergence-form operators with uniformly elliptic coefficients. Our intention is to motivate some ideas and concepts of the operator-theoretic framework that we develop in the present paper. To fix ideas, let $Q \subset \mathbb{R}^{n}$ denote an open, bounded domain, $f \in L^{2}(Q)$, and let $u_{\varepsilon} \in H_{0}^{1}(Q)$ denote the unique weak solution to

$$
-\nabla \cdot\left(a(\dot{\bar{\varepsilon}}) \nabla u_{\varepsilon}\right)=f \quad \text { in } Q
$$

where $a: \mathbb{R}^{n} \rightarrow \mathbb{R}^{n \times n}$ denotes a uniformly elliptic coefficient field, i.e., $a: \mathbb{R}^{n} \rightarrow \mathbb{R}_{\lambda, \Lambda}^{n \times n}$ is measurable and $\mathbb{R}_{\lambda, \Lambda}^{n \times n}$ denotes (for some fixed constants of ellipticity $0<\lambda \leqslant \Lambda$ ) the set of matrices $a_{0} \in \mathbb{R}^{n \times n}$ satisfying $a_{0} \xi \cdot \xi \geqslant \lambda|\xi|^{2}$ and $\left|a_{0} \xi\right| \leqslant \Lambda|\xi|$ (for all $\xi \in \mathbb{R}^{n}$ ).

Periodic homogenization is concerned with the case that the coefficient field $a$ in (3) is periodic, say $a(\cdot+k)=a(\cdot)$ a.e. in $\mathbb{R}^{n}$ for all $k \in \mathbb{Z}^{n}$. Under this condition a classical result (e.g., $\left.[21,3,10]\right)$ states that there exists a uniformly elliptic matrix $a_{\text {hom }} \in \mathbb{R}^{n \times n}$ such that $\left(u_{\varepsilon}\right)_{\varepsilon>0}$ weakly converges in $H_{0}^{1}(Q)$ (as $\varepsilon \rightarrow 0$ ) to the unique weak solution $u \in H_{0}^{1}(Q)$ to the homogenized equation

$$
-\nabla \cdot\left(a_{\mathrm{hom}} \nabla u\right)=f
$$

The homogenized coefficient matrix $a_{\text {hom }}$ is characterized by the formula

$$
a_{\mathrm{hom}} e_{i}=\int_{\square} a\left(\nabla \varphi_{i}+e_{i}\right) d x \quad(i \in\{1, \ldots, n\}),
$$


where $\square:=[0,1)^{n}$ represents the reference cell of periodicity, $e_{i} \in \mathbb{R}^{n}$ denotes the $i$ 'th unit vector, and $\varphi_{i} \in H_{\mathrm{loc}}^{1}\left(\mathbb{R}^{n}\right)$ denotes a periodic solution to the corrector equation

$$
-\nabla \cdot\left(a\left(\nabla \varphi_{i}+e_{i}\right)\right)=0 \quad \text { in } \mathbb{R}^{n} .
$$

Thanks to the periodicity of $a,(4)$ can be solved by lifting the equation to the Sobolev space of periodic functions with zero mean (where Poincaré's inequality and uniform ellipticity of $a$ implies coercivity of the elliptic operator $-\nabla \cdot(a \nabla)$ ). The corrector equation (4) is a key object in homogenization theory for elliptic equations, since its solution - the corrector $\varphi_{i}$ - captures the spatial oscillations of $u_{\varepsilon}$ induced by the heterogeneity of the coefficient field $a$. This can be expressed in terms of the (formal) asymptotic expansions (using Einstein's summation convention)

$$
u_{\varepsilon}(x) \approx u(x)+\varepsilon \varphi_{i}\left(\frac{x}{\varepsilon}\right) \partial_{i} u(x), \quad \nabla u_{\varepsilon}(x) \approx \nabla u(x)+\nabla \varphi_{i}\left(\frac{x}{\varepsilon}\right) \partial_{i} u(x)
$$

or (more precisely) in form of the two-scale convergence statement

$$
\nabla u_{\varepsilon} \stackrel{2}{\rightarrow} \nabla u+\nabla \varphi_{i} \partial_{i} u
$$

where $\stackrel{2}{\rightarrow}$ denotes the notion of (strong) two-scale convergence introduced in [24] and further investigated in [1] (see also [18]) and $\nabla u+\nabla \varphi_{i} \partial_{i} u$ denotes the function $(x, y) \mapsto \nabla u(x)+\nabla \varphi_{i}(y) \partial_{i} u(x)$. Also, another method closely related to two-scale convergence is the periodic unfolding method $[8,39,9]$, which is based on an unfolding operator that equivalently characterizes two-scale convergence (see also [20]).

In the stochastic case the coefficient field $a$ is assumed to be a random object, i.e., $(a(x))_{x \in \mathbb{R}^{n}}$ is viewed as a family of $\mathbb{R}_{\lambda, \Lambda}^{n \times n}$-valued random variables. Minimial requirements for stochastic homogenization (towards a deterministic limit) are stationarity and ergodicity. The former means that for any $x_{1}, \ldots, x_{N} \in \mathbb{R}^{n}$ and $z \in \mathbb{R}^{n}$, the distribution of $\left(a\left(x_{1}+z\right), \ldots, a\left(x_{N}+z\right)\right)$ is independent of the shift $z \in \mathbb{R}^{n}$. Ergodicity means that any shift-invariant (measurable) subset of the coefficients has probability 1 or 0 . In their seminal work [25], Papanicolaou and Varadhan rephrased these conditions in an analytic framework that by now became a standard in stochastic homogenization. In the following we recall their framework and some objects involved in their approach. We refer to $[25,15]$ for details (see also the lecture notes [22] for a self-contained presentation). The idea is to equip $\Omega:=\left\{a: \mathbb{R}^{n} \rightarrow \mathbb{R}_{\lambda, \Lambda}^{n \times n}: a\right.$ measurable $\}$, the set of all uniformly elliptic coefficient fields, with a probability measure $\mu$ and to draw $a$ randomly from $\Omega$ according to $\mu$. The precise setup is as follows:

Assumption 1. Let $(\Omega, \Sigma, \mu)$ denote a probability space with a countably generated $\sigma$-algebra (that implies separability of $L^{2}(\Omega)$ ), and let $\tau=\left\{\tau_{x}: x \in \mathbb{R}^{n}\right\}$ be a group of measurable bijections $\tau_{x}: \Omega \rightarrow \Omega$ such that:

(a) (Group property). $\tau_{0}=\operatorname{id}_{\Omega}$ and $\tau_{x+y}=\tau_{x} \circ \tau_{y}$ for all $x, y \in \mathbb{R}^{n}$.

(b) (Measure preservation). $\mu\left(\tau_{x} A\right)=\mu(A)$ for all $A \in \Sigma$ and $x \in \mathbb{R}^{n}$.

(c) (Measurability). $(\omega, x) \mapsto \tau_{x} \omega$ is $\left(\Sigma \otimes \mathcal{L}\left(\mathbb{R}^{n}\right), \Sigma\right)$-measurable $\left(\mathcal{L}\left(\mathbb{R}^{n}\right)\right.$ denotes the Lebesgue- $\sigma$ algebra on $\left.\mathbb{R}^{n}\right)$.

Let $a_{0}: \Omega \rightarrow \mathbb{R}_{\lambda, \Lambda}^{n \times n}$ be measurable, then $a(x, \omega):=a_{0}\left(\tau_{x} \omega\right)$ defines a uniformly elliptic, random coefficient field that is stationary (thanks to property (b)). Moreover, in this framework, the assumption of ergodicity reads: any shift-invariant set $A \in \Sigma$ (i.e., $\tau_{x} A \subset A$ for all $x \in \mathbb{R}^{n}$ ) satisfies $\mu(A) \in\{0,1\}$. 
Under the conditions of Assumption 1 and ergodicity, Papanicolaou and Varadhan proved the following homogenization result: For $f \in L^{2}(Q)$ and $\varepsilon>0$, let $u_{\varepsilon} \in H_{0}^{1}(Q) \otimes L^{2}(\Omega)$ denote the unique (Lax-Milgram) solution to

$$
-\nabla \cdot\left(a_{0}\left(\tau_{\frac{x}{\varepsilon}} \omega\right) \nabla u_{\varepsilon}(x, \omega)\right)=f(x) \quad \text { in } Q \times \Omega .
$$

Then there exists a uniformly elliptic coefficient matrix $a_{\text {hom }} \in \mathbb{R}^{n \times n}$ (only depending on $\mu$ and $\left.a_{0}\right)$ such that $u_{\varepsilon}$ weakly converges in $H_{0}^{1}(Q) \otimes L^{2}(\Omega)$ to the unique solution $u \in H_{0}^{1}(Q)$ of the homogenized equation $-\nabla \cdot\left(a_{\text {hom }} \nabla u\right)=f$ in $Q$. The proof of Papanicolaou and Varadhan is based on Tartar's method of oscillating test functions and the main difficulty is to give sense to the corrector equation (4) and the corrector $\varphi_{i}$ in the stochastic case.

An alternative proof of the above homogenization result was introduced in [4], based on a stochastic counterpart of two-scale convergence - the notion of stochastic two-scale convergence in the mean (see also [2]). In particular, a bounded sequence $\left(u_{\varepsilon}\right)_{\varepsilon}$ in $L^{2}(Q) \otimes L^{2}(\Omega)$ is said to twoscale converge in the mean to a function $u \in L^{2}(Q) \otimes L^{2}(\Omega)$, if for all test functions $\eta \in C_{c}^{\infty}(Q)$ and $\varphi \in L^{2}(\Omega)$,

$$
\int_{\Omega} \int_{Q} u_{\varepsilon}(x, \omega) \eta(x) \varphi\left(\tau_{\frac{x}{\varepsilon}} \omega\right) d x d \mu(\omega) \rightarrow \int_{\Omega} \int_{Q} u(x, \omega) \eta(x) \varphi(\omega) d x d \mu(\omega) .
$$

Stochastic homogenization via unfolding. Recently, in $[23,14]$ Heida and the first two authors reconsidered the notion of two-scale convergence in the mean from the perspective of an unfolding operator (see also [38]). This approach is motivated by (and shares many similarities with) the well-established notion of periodic unfolding [8]. In the following we briefly recall its definition (for more detail, see [14]).

For $\eta \in L^{2}(Q)$ and $\varphi \in L^{2}(\Omega)$, we define

$$
\mathcal{T}_{\varepsilon}(\eta \otimes \varphi)(x, \omega)=\eta(x) \varphi\left(\tau_{-\frac{x}{\varepsilon}} \omega\right) .
$$

Using the measure preserving property from Assumption 1 (b), it follows that

$$
\left\|\mathcal{T}_{\varepsilon}(\eta \otimes \varphi)\right\|_{L^{2}(Q) \otimes L^{2}(\Omega)}=\|\eta \otimes \varphi\|_{L^{2}(Q) \otimes L^{2}(\Omega)},
$$

and by the density of the linear span of simple tensor products

$$
L^{2}(Q) \stackrel{a}{\otimes} L^{2}(\Omega):=\operatorname{lin}\left\{(x, \omega) \mapsto \varphi(x) \psi(\omega) ; \varphi \in L^{2}(Q), \psi \in L^{2}(\Omega)\right\} \subset L^{2}(Q) \otimes L^{2}(\Omega) \text { dense, }
$$

$\mathcal{T}_{\varepsilon}$ extends to a linear isometry $\mathcal{T}_{\varepsilon}: L^{2}(Q) \otimes L^{2}(\Omega) \rightarrow L^{2}(Q) \otimes L^{2}(\Omega)$. Moreover, the equality $\mathcal{T}_{\varepsilon} \mathcal{T}_{-\varepsilon}=1$ on simple tensors implies that $\mathcal{T}_{\varepsilon}$ is in fact unitary. A simple consequence of this definition is that for a bounded sequence $u_{\varepsilon} \in L^{2}(Q) \otimes L^{2}(\Omega)$ the weak convergence $\mathcal{T}_{\varepsilon} u_{\varepsilon} \rightarrow u$ is equivalent to stochastic two-scale convergence in the mean of $u_{\varepsilon}$ to $u$ (in sense of (6)). Also, note that using the stochastic unfolding operator, we might rephrase equation (5) as

$$
-\nabla \cdot\left(\mathcal{T}_{-\varepsilon} a_{0} \mathcal{T}_{\varepsilon} \nabla u\right)=f .
$$

In the present paper we go one step further and reconsider the idea of unfolding on an abstract operator-theoretic level where

- $L^{2}(Q)$ and $L^{2}(\Omega)$ are replaced by general Hilbert spaces $H_{d}$ and $H_{s}$, 
- the stochastic unfolding operator is replaced by a family of well-structured unitary operators,

- $-\nabla \cdot$ and $\nabla$ are replaced by densely defined closed (unbounded) linear operators $C_{d}^{*}$ and $C_{d}$.

Our motivation is to develop a unified, operator-theoretic approach to homogenization (in the mean) of linear evolutionary problems with periodic, quasiperiodic or random (stationary) coefficients. The abstract unfolding strategy that we propose applies to a variety of linear PDEs and we present some examples in Section 5. Here we briefly explain one of the examples - stochastic homogenization of Maxwell's equations, which we discuss in detail in Section 5.3. In particular, for $Q \subseteq \mathbb{R}^{3}$ open, we consider the following system of equations: Find $\left(u_{\varepsilon}, q_{\varepsilon}\right): \mathbb{R} \times Q \rightarrow \mathbb{C}^{3} \times \mathbb{C}^{3}$ such that

$$
\begin{array}{r}
\partial_{t}\left(\eta_{\varepsilon} u_{\varepsilon}\right)+\sigma_{\varepsilon} u_{\varepsilon}-\operatorname{curl} q_{\varepsilon}=f, \\
\partial_{t}\left(\mu_{\varepsilon} q_{\varepsilon}\right)+\operatorname{curl} u_{\varepsilon}=g,
\end{array}
$$

where $(f, g): \mathbb{R} \rightarrow L^{2}(Q)^{3} \oplus L^{2}(Q)^{3}$ is a datum and the random, oscillating coefficients are given in the form $\eta_{\varepsilon}(x, \omega)=\eta_{0}\left(x, \tau_{\frac{x}{\varepsilon}} \omega\right), \sigma_{\varepsilon}(x, \omega)=\sigma_{0}\left(x, \tau_{\underline{\varepsilon}} \omega\right), \mu_{\varepsilon}(x, \omega)=\mu_{0}\left(x, \tau_{\underline{\varepsilon}} \omega\right)$ with $\eta_{0}, \sigma_{0}, \mu_{0} \in$ $L^{\infty}(Q \times \Omega)^{3 \times 3}$ that satisfy suitable assumptions. Note that the solution depends on $\omega \in \Omega$, which we see as a random configuration of the medium, and therefore we view the solution also as a random field, i.e., we seek functions such that at (almost) each time instance $t \in \mathbb{R}$ satisfy $\left(u_{\varepsilon}(t), q_{\varepsilon}(t)\right) \in\left(L^{2}(Q) \otimes L^{2}(\Omega)\right)^{3} \oplus\left(L^{2}(Q) \otimes L^{2}(\Omega)\right)^{3}$. In fact, we phrase (9) in the form of the operator equation (1) given on the functional space $L_{\nu}^{2}\left(\mathbb{R} ;\left(L^{2}(Q) \otimes L^{2}(\Omega)\right)^{3} \oplus\left(L^{2}(Q) \otimes L^{2}(\Omega)\right)^{3}\right)$, which is an exponentially weighted $L^{2}$-space with a parameter $\nu \in \mathbb{R}$ (see Section 4.1). In the limit $\varepsilon \rightarrow 0$, we derive a two-scale homogenized system, that in the case of ergodic coefficients reads: Find $\left(u_{0}, \chi_{1}, q_{0}, \chi_{2}\right) \in L_{\nu}^{2}\left(\mathbb{R} ; L^{2}(Q)^{3} \oplus\left(L^{2}(Q) \otimes L_{\text {pot }}^{2}(\Omega)\right) \oplus L^{2}(Q)^{3} \oplus\left(L^{2}(Q) \otimes L_{\text {pot }}^{2}(\Omega)\right)\right)$ such that

$$
\begin{array}{r}
\partial_{t}\left(\mathbb{E}\left[\eta_{0}\left(u_{0}+\chi_{1}\right)\right]\right)+\mathbb{E}\left[\sigma_{0}\left(u_{0}+\chi_{1}\right)\right]-\operatorname{curl} q_{0}=f, \\
\partial_{t}\left(\mathbb{E}\left[\mu_{0}\left(q_{0}+\chi_{2}\right)\right]\right)+\operatorname{curl} u_{0}=g, \\
-\operatorname{div}_{\omega}\left(\partial_{t}\left(\eta_{0}\left(u_{0}+\chi_{1}\right)\right)+\sigma_{0}\left(u_{0}+\chi_{1}\right)\right)=0 \\
-\operatorname{div}_{\omega}\left(\partial_{t}\left(\mu_{0}\left(q_{0}+\chi_{2}\right)\right)\right)=0,
\end{array}
$$

where $\mathbb{E}[\cdot]$ denotes the mathematical expectation in $(\Omega, \Sigma, \mu)$ and $\operatorname{div}_{\omega}$ is the stochastic divergence, which is defined in Section 5.1. We may view the first two equations as the effective Maxwell system for the deterministic variables $\left(u_{0}, q_{0}\right)$ and the correctors $\left(\chi_{1}, \chi_{2}\right)$, that account for the microstructure evolution in the material, are determined by the last two corrector equations, cf. Remark 5.12. In particular, we obtain that, as $\varepsilon \rightarrow 0$,

$$
\mathcal{T}_{\varepsilon}\left(u_{\varepsilon}, q_{\varepsilon}\right) \rightarrow\left(u_{0}+\chi_{1}, q_{0}+\chi_{2}\right) \quad \text { weakly in } L_{\nu}^{2}\left(\mathbb{R} ;\left(L^{2}(Q) \otimes L^{2}(\Omega)\right)^{3} \oplus\left(L^{2}(Q) \otimes L^{2}(\Omega)\right)^{3}\right) .
$$

A similar result in a periodic-stochastic setting has been obtained in [33]. With our operatortheoretic approach we are able to dispose of the continuity condition on the coefficients in the slow variable. Next, we can treat highly oscillatory mixed type equations by only requiring the sum of $\sigma_{0}$ and $\eta_{0}$ to be positive. Moreover, for suitably regular right-hand sides $(f, g)$, we obtain the following corrector result:

$$
\left\|u_{\varepsilon}-\mathcal{T}_{-\varepsilon} \chi_{1}-u_{0}\right\|_{L_{\nu}^{2}\left(\mathbb{R} ; L^{2}(Q) \otimes L^{2}(\Omega)^{3}\right)}^{2}+\left\|q_{\varepsilon}-\mathcal{T}_{-\varepsilon} \chi_{2}-q_{0}\right\|_{L_{\nu}^{2}\left(\mathbb{R} ; L^{2}(Q) \otimes L^{2}(\Omega)^{3}\right)}^{2} \rightarrow 0 \quad \text { as } \varepsilon \rightarrow 0 .
$$

See Section 5.3 for details. 


\section{The operator-theoretic setting for unfolding}

In this section we introduce the setting for abstract two-scale convergence and provide some compactness results which will be useful in the following sections. Throughout this section, we let $H_{d}$ and $H_{s}$ be Hilbert spaces, $m, n \in \mathbb{N}$; ' $d$ ' and ' $s$ ' are a reminder of 'deterministic' and 'stochastic'.

Abstract "differential" operators. We consider densely defined closed linear operators

$$
C_{d}: \operatorname{dom}\left(C_{d}\right) \subseteq H_{d}^{m} \rightarrow H_{d}^{n}, \quad C_{s}: \operatorname{dom}\left(C_{s}\right) \subseteq H_{s}^{m} \rightarrow H_{s}^{n} .
$$

Given a Hilbert space $H$ the canonical extension of $C_{d}$ to operators from the Hilbert space tensor product $H_{d}^{m} \otimes H$ to $H_{d}^{n} \otimes H$ will be - as a rule - again denoted by $C_{d}$; and similarly for $C_{s}$. In the applications, we have in mind, we extend $C_{d}$ to the space $H_{d}^{m} \otimes H_{s}$ attaining values in $H_{d}^{n} \otimes H_{s}$ and $C_{s}$ as closed, densely defined linear operator from $H_{d} \otimes H_{s}^{m}$ to $H_{d} \otimes H_{s}^{n}$. Note that we will further identify $H_{d}^{m} \otimes H_{s}=\left(H_{d} \otimes H_{s}\right)^{m}=H_{d} \otimes H_{s}^{m}$. Thus, the extended operators $C_{d}$ and $C_{s}$ are both operators defined on (subsets of) $\left(H_{d} \otimes H_{s}\right)^{m}$ with values in $\left(H_{d} \otimes H_{s}\right)^{n}$. With this in mind, we require the following compatibility conditions for $C_{s}$ and $C_{d}$ :

$$
\begin{aligned}
& \exists D_{1} \subseteq H_{d} \text { dense } \forall x \in D_{1}, y \in H_{s}^{m}: x \otimes y \in \operatorname{dom}\left(C_{d}\right), \\
& \exists D_{2} \subseteq H_{d} \text { dense } \forall x \in D_{2}, y \in H_{s}^{n}: x \otimes y \in \operatorname{dom}\left(C_{d}^{*}\right), \\
& \exists E_{1} \subseteq H_{s} \text { dense } \forall y \in E_{1}, x \in H_{d}^{m}: x \otimes y \in \operatorname{dom}\left(C_{s}\right), \\
& \exists E_{2} \subseteq H_{s} \text { dense } \forall y \in E_{2}, x \in H_{d}^{n}: x \otimes y \in \operatorname{dom}\left(C_{s}^{*}\right) .
\end{aligned}
$$

The first and third conditions are trivially satisfied, if $m=1$, the second and fourth if $n=1$. In applications discussed later on, $D_{1}=D_{2}=C_{c}^{\infty}(Q)$ for some open $Q \subseteq \mathbb{R}^{n}$ (a similar choice can be made for $\left.E_{1}, E_{2}\right)$.

Remark 2.1. Note the following consequence of our notation convention. Given a bounded linear operator $T \in L(H, K)$ for $H, K$ Hilbert spaces, we have $T C_{d} \subseteq C_{d} T$, and $T C_{s} \subseteq C_{s} T$, see also Lemma 2.5 below.

Unfolding family. We call a strongly continuous map $\mathcal{T}: \mathbb{R} \backslash\{0\} \rightarrow L\left(H_{d} \otimes H_{s}\right)$ taking values in the set of unitary operators an unfolding family, if the following structural hypotheses are satisfied (where the action of $\mathcal{T}_{\varepsilon}$ on powers of $H_{d} \otimes H_{s}$ is understood "component-wise", re-using the notation):

$$
\begin{aligned}
\mathcal{T}_{\varepsilon}=\mathcal{T}_{-\varepsilon}^{-1} & (\varepsilon \in \mathbb{R} \backslash\{0\}), \\
\varepsilon C_{d} \mathcal{T}_{-\varepsilon} \varphi=\varepsilon \mathcal{T}_{-\varepsilon} C_{d} \varphi+C_{s} \mathcal{T}_{-\varepsilon} \varphi & \left(\varphi \in \operatorname{dom}\left(C_{d}\right) \cap \operatorname{dom}\left(C_{s}\right) \subseteq\left(H_{d} \otimes H_{s}\right)^{m}, \varepsilon \in \mathbb{R} \backslash\{0\}\right), \\
\mathcal{T}_{\varepsilon} C_{s} \subseteq C_{s} \mathcal{T}_{\varepsilon} & (\varepsilon \in \mathbb{R} \backslash\{0\}), \\
\mathcal{T}_{\varepsilon} v=v & \left(v \in \operatorname{ker}\left(C_{s}\right), \varepsilon \in \mathbb{R} \backslash\{0\}\right) .
\end{aligned}
$$

We remark here that (12) (in conjunction with (10)) implies,

$$
\varepsilon C_{d}^{*} \mathcal{T}_{-\varepsilon} \varphi=\varepsilon \mathcal{T}_{-\varepsilon} C_{d}^{*} \varphi+\mathcal{T}_{-\varepsilon} C_{s}^{*} \varphi \quad\left(\varphi \in \operatorname{dom}\left(C_{s}^{*}\right) \cap \operatorname{dom}\left(C_{d}^{*}\right) \subseteq\left(H_{d} \otimes H_{s}\right)^{n}, \varepsilon \in \mathbb{R} \backslash\{0\}\right) .
$$

The latter will be particularly important, when we discuss time-dependent problems. 
Remark 2.2. The stochastic unfolding operator introduced in Section 1.1 together with $C_{d}=\nabla$, and $C_{s}$ denoting the stochastic gradient, satisfies the above assumptions (see Section 5).

Within the above setting we define (stochastic) 2-scale convergence as follows.

Definition 2.3. Let $\left(u_{\varepsilon}\right)_{\varepsilon>0}$ in $H_{d} \otimes H_{s}$. Then $\left(u_{\varepsilon}\right)_{\varepsilon}$ is said to strongly (weakly) 2-scale converge to $u \in H_{d} \otimes H_{s}$ (we also use the notation $u_{\varepsilon} \stackrel{2}{\rightarrow} u\left(u_{\varepsilon} \stackrel{2}{\rightarrow} u\right)$ ), if

$$
\mathcal{T}_{\varepsilon} u_{\varepsilon} \rightarrow u \quad\left(\mathcal{T}_{\varepsilon} u_{\varepsilon} \rightarrow u\right)
$$

strongly (weakly) as $\varepsilon \rightarrow 0$.

Remark 2.4. Strictly speaking 2 -scale convergence is only defined for families $\left(u_{\varepsilon}\right)_{\varepsilon>0}$. In the following, we will however also say that "a subsequence of $\left(u_{\varepsilon}\right)_{\varepsilon>0}$ (weakly/strongly) 2-scale converges to some $u$ ". By this, we mean that there exists a sequence $\left(\varepsilon_{k}\right)_{k}$ in $(0, \infty)$ converging to 0 such that $\mathcal{T}_{\varepsilon_{k}} u_{\varepsilon_{k}} \rightarrow u$ weakly as $k \rightarrow \infty$. As the particular subsequence will not be important in the considerations we are aiming for, we shall however re-use $\varepsilon$ and dispense with $k$.

In the following we establish various properties that we shall exploit in our abstract homogenization scheme, and highlight analogies to stochastic two-scale convergence in the mean and periodic unfolding, respectively. We begin with an abstract counter part of [4, Theorem 3.7]. In order to avoid cluttered notation as much as possible, we often write $H_{d} \otimes H_{s}$ regardless of the number of components of the objects under consideration. We begin with an auxiliary result.

Unless explicitly stated otherwise, we shall not assume condition (14) in this section. The conditions (10)-(13), however, are assumed to be in effect.

Lemma 2.5. Let $P$ be the orthogonal projection onto $\operatorname{ker}\left(C_{s}\right) \subseteq\left(H_{d} \otimes H_{s}\right)^{m}$. Then $P \mathcal{T}_{\varepsilon}=\mathcal{T}_{\varepsilon} P$ for all $\varepsilon \in \mathbb{R} \backslash\{0\}$.

Proof. Let $\varepsilon \in \mathbb{R} \backslash\{0\}$. Then we have $\mathcal{T}_{\varepsilon} C_{s} \subseteq C_{s} \mathcal{T}_{\varepsilon}$ by (13). Since $\mathcal{T}_{\varepsilon}^{*}=\mathcal{T}_{-\varepsilon}$ by (11), we have $C_{s}^{*} \mathcal{T}_{-\varepsilon}=\left(\mathcal{T}_{\varepsilon} C_{s}\right)^{*} \supseteq\left(C_{s} \mathcal{T}_{\varepsilon}\right)^{*}=\mathcal{T}_{-\varepsilon} C_{s}^{*}$. Hence, we deduce for all $\varepsilon \in \mathbb{R} \backslash\{0\}$

$$
\mathcal{T}_{\varepsilon} C_{s}^{*} C_{s} \subseteq C_{s}^{*} \mathcal{T}_{\varepsilon} C_{s} \subseteq C_{s}^{*} C_{s} \mathcal{T}_{\varepsilon}
$$

Since $C_{s}^{*} C_{s}$ is self-adjoint and $\mathcal{T}_{\varepsilon}$ continuous, we deduce that for all bounded, measurable functions $f: \sigma\left(C_{s}^{*} C_{s}\right) \rightarrow \mathbb{R}, \mathcal{T}_{\varepsilon} f\left(C_{s}^{*} C_{s}\right)=f\left(C_{s}^{*} C_{s}\right) \mathcal{T}_{\varepsilon}$. In particular, $f=\chi_{\{0\}}$ is a possible choice. $\chi_{\{0\}}\left(C_{s}^{*} C_{s}\right)$ is the orthogonal projection onto $\operatorname{ker}\left(C_{s}^{*} C_{s}\right)$. Note that $\operatorname{ker}\left(C_{s}^{*} C_{s}\right)=\operatorname{ker}\left(C_{s}\right)$. Indeed, $\operatorname{ker}\left(C_{s}\right) \subseteq$ $\operatorname{ker}\left(C_{s}^{*} C_{s}\right)$ is trivial; for $\varphi \in \operatorname{ker}\left(C_{s}^{*} C_{s}\right)$, we test the equation $C_{s}^{*} C_{s} \varphi=0$ with $\varphi \in \operatorname{ker}\left(C_{s}^{*} C_{s}\right) \subseteq$ $\operatorname{dom}\left(C_{s}^{*} C_{s}\right) \cap \operatorname{dom}\left(C_{s}\right)$ to obtain $\left\langle C_{s} \varphi, C_{s} \varphi\right\rangle=0$, that is, $\operatorname{ker}\left(C_{s}\right) \supseteq \operatorname{ker}\left(C_{s}^{*} C_{s}\right)$. We infer $P=$ $\chi_{\{0\}}\left(C_{s}^{*} C_{s}\right)$, which yields the assertion.

Lemma 2.6. Let $K \subseteq H_{s}$ be a closed subspace and $P$ the projection onto $K$. Then $P C_{d} \subseteq C_{d} P$.

Proof. We have identified $C_{d}$ with $C_{d} \otimes 1_{H_{s}}$ and $P$ with $1_{H_{d}} \otimes P$. Thus, the assertion follows from standard tensor-product theory of operators in Hilbert spaces, see, e.g., [40, Appendix].

Lemma 2.7. Let $\left(u_{\varepsilon}\right)_{\varepsilon}$ and $\left(\varepsilon C_{d} u_{\varepsilon}\right)_{\varepsilon}$ be bounded in $\left(H_{d} \otimes H_{s}\right)^{m}$ and $\left(H_{d} \otimes H_{s}\right)^{n}$, respectively. Then there are subsequences of $\left(u_{\varepsilon}\right)_{\varepsilon}$ and $\left(\varepsilon C_{d} u_{\varepsilon}\right)_{\varepsilon}$ as well as $u \in \operatorname{dom}\left(C_{s}\right)$ such that $\left(u_{\varepsilon}\right)_{\varepsilon}$ weakly 2-scale converges to $u$ and $\left(\varepsilon C_{d} u_{\varepsilon}\right)_{\varepsilon}$ weakly 2-scale converges to $C_{s} u$. 
Proof. There exist subsequences such that $\left(\mathcal{T}_{\varepsilon} u_{\varepsilon}\right)_{\varepsilon}$ weakly converges to $u \in\left(H_{d} \otimes H_{s}\right)^{m}$ and $\left(\varepsilon \mathcal{T}_{\varepsilon} C_{d} u_{\varepsilon}\right)_{\varepsilon}$ weakly converges to $v \in\left(H_{d} \otimes H_{s}\right)^{n}$, respectively. We will show that $u \in \operatorname{dom}\left(C_{s}\right)$ and $C_{s} u=v$. For this, we let $g \in \operatorname{dom}\left(C_{s}^{*}\right) \cap \operatorname{dom}\left(C_{d}^{*}\right)$ and compute (using (15))

$$
\begin{aligned}
\left\langle\varepsilon \mathcal{T}_{\varepsilon} C_{d} u_{\varepsilon}, g\right\rangle & =\left\langle u_{\varepsilon}, \varepsilon C_{d}^{*} \mathcal{T}_{-\varepsilon} g\right\rangle \\
& =\left\langle u_{\varepsilon}, \varepsilon \mathcal{T}_{-\varepsilon} C_{d}^{*} g+\mathcal{T}_{-\varepsilon} C_{s}^{*} g\right\rangle \\
& =\left\langle\varepsilon \mathcal{T}_{\varepsilon} u_{\varepsilon}, C_{d}^{*} g\right\rangle+\left\langle\mathcal{T}_{\varepsilon} u_{\varepsilon}, C_{s}^{*} g\right\rangle \\
& \rightarrow\left\langle u, C_{s}^{*} g\right\rangle .
\end{aligned}
$$

Since $\operatorname{dom}\left(C_{s}^{*}\right) \cap \operatorname{dom}\left(C_{d}^{*}\right)$ is an operator core for $C_{s}^{*}$ by (10), we obtain $u \in \operatorname{dom}\left(C_{s}\right)$ and $C_{s} u=$ $(\mathrm{w}-) \lim _{\varepsilon \rightarrow 0} \varepsilon \mathcal{T}_{\varepsilon} C_{d} u_{\varepsilon}$.

Theorem 2.8 (Compactness). Assume (10)-(13).

(a) Let $\left(u_{\varepsilon}\right)_{\varepsilon}$ be uniformly bounded in $\operatorname{dom}\left(C_{d}\right)$. Assume that $\left(u_{\varepsilon}\right)_{\varepsilon}$ and $\left(C_{d} u_{\varepsilon}\right)_{\varepsilon}$ weakly 2scale converge. Then there exist $u \in \operatorname{dom}\left(C_{d}\right) \cap \operatorname{ker}\left(C_{s}\right)$ and $v \in \overline{\operatorname{ran}}\left(C_{s}\right)$ such that $u_{\varepsilon} \stackrel{2}{\rightarrow} u$ and $C_{d} u_{\varepsilon} \stackrel{2}{\rightarrow} C_{d} u+v$.

(b) Let $\left(q_{\varepsilon}\right)_{\varepsilon}$ be uniformly bounded in $\operatorname{dom}\left(C_{d}^{*}\right)$. Assume that $\left(q_{\varepsilon}\right)_{\varepsilon}$ and $\left(C_{d}^{*} q_{\varepsilon}\right)_{\varepsilon}$ weakly 2scale converge. Then there exist $q \in \operatorname{dom}\left(C_{d}^{*}\right) \cap \operatorname{ker}\left(C_{s}^{*}\right)$ and $w \in \overline{\operatorname{ran}}\left(C_{s}^{*}\right)$ such that $q_{\varepsilon} \stackrel{2}{\rightarrow} q$ and $C_{d}^{*} q_{\varepsilon} \stackrel{2}{\rightarrow} C_{d}^{*} q+w$.

(c) Assume, in addition, $m=1$ and that (14) holds. Let $\left(u_{\varepsilon}\right)_{\varepsilon}$ be uniformly bounded in $\operatorname{dom}\left(C_{d}\right)$. Then there exists $u \in \operatorname{dom}\left(C_{d}\right) \cap \operatorname{ker}\left(C_{s}\right)$ and $v \in \overline{\operatorname{ran}}\left(C_{s}\right) \cap \overline{\operatorname{ran}}\left(C_{s}^{*}\right)^{n} \subseteq\left(H_{d} \otimes H_{s}\right)^{n}$ such that (a subsequence of $\left(u_{\varepsilon}\right)_{\varepsilon}$ weakly 2-scale converges to $u$ and $\left(C_{d} u_{\varepsilon}\right)_{\varepsilon}$ weakly 2-scale converges to $C_{d} u+v$.

Proof. (a) For suitable subsequences, denote by $u$ and $\bar{v}$ the corresponding weak 2-scale-limits of $\left(u_{\varepsilon}\right)_{\varepsilon}$ and $\left(C_{d} u_{\varepsilon}\right)_{\varepsilon}$. By Lemma 2.7, we deduce that $u \in \operatorname{ker}\left(C_{s}\right)$. By weak continuity of $P$, it is easy to see that $\left(P \mathcal{T}_{\varepsilon} u_{\varepsilon}\right)_{\varepsilon}$ weakly converges to $P u=u$. By Lemma 2.5 , we have $P \mathcal{T}_{\varepsilon}=\mathcal{T}_{\varepsilon} P$. Thus, since $P C_{d} \subseteq C_{d} P$, we get $P u_{\varepsilon} \in \operatorname{dom}\left(C_{d}\right) \cap \operatorname{ker}\left(C_{s}\right) \subseteq \operatorname{dom}\left(C_{d}\right) \cap \operatorname{dom}\left(C_{s}\right)$ and so, by (12),

$$
C_{d} P \mathcal{T}_{\varepsilon} u_{\varepsilon}=\mathcal{T}_{\varepsilon} C_{d} P u_{\varepsilon}=\mathcal{T}_{\varepsilon} P C_{d} u_{\varepsilon}
$$

Thus, since the right-hand side is uniformly bounded in $\varepsilon>0$, so is the left-hand side. From the closedness of $C_{d} P$, it follows that $u=P u \in \operatorname{dom}\left(C_{d}\right)$.

We define $v:=\bar{v}-C_{d} u$. Then we compute for suitable $\varepsilon>0$ and all $w \in \operatorname{ker}\left(C_{s}^{*}\right) \cap \operatorname{dom}\left(C_{d}^{*}\right)$ :

$$
\left\langle\mathcal{T}_{\varepsilon} C_{d} u_{\varepsilon}, w\right\rangle=\left\langle\mathcal{T}_{\varepsilon} u_{\varepsilon}, C_{d}^{*} w\right\rangle
$$

Letting $\varepsilon \rightarrow 0$ on both sides, we obtain

$$
\left\langle C_{d} u+v, w\right\rangle=\left\langle C_{d} u, w\right\rangle
$$

which leads to

$$
\langle v, w\rangle=0 \quad\left(w \in \operatorname{ker}\left(C_{s}^{*}\right) \cap \operatorname{dom}\left(C_{d}^{*}\right)\right) .
$$

Since $\operatorname{ker}\left(C_{s}^{*}\right) \cap \operatorname{dom}\left(C_{d}^{*}\right)$ is dense in $\operatorname{ker}\left(C_{s}^{*}\right)$ by $(10)$, we infer $v \in \operatorname{ker}\left(C_{s}^{*}\right)^{\perp}=\overline{\operatorname{ran}}\left(C_{s}\right)$. (a).

(b) By symmetry of the conditions (10)-(13) in $C_{d}$ and $C_{d}^{*}$, the proof follows analogously to 
(c) Choose subsequences of $\left(\mathcal{T}_{\varepsilon} u_{\varepsilon}\right)_{\varepsilon}$ and $\left(\mathcal{T}_{\varepsilon} C_{d} u_{\varepsilon}\right)_{\varepsilon}$ that weakly converge to some $u$ and $\bar{v}$. By Lemma 2.7, we deduce that $C_{s} u=\left(\mathrm{w}_{-}\right) \lim _{\varepsilon \rightarrow 0} \varepsilon \mathcal{T}_{\varepsilon} C_{d} u_{\varepsilon}=0 \cdot \bar{v}$, which yields $u \in \operatorname{ker}\left(C_{s}\right)$. Moreover, we may choose a weakly convergent subsequence of $\left(u_{\varepsilon}\right)_{\varepsilon}$ in $\operatorname{dom}\left(C_{d}\right)$. Denote the limit by $\tilde{u}$. Let $P$ be the orthogonal projection onto $\operatorname{ker}\left(C_{s}\right)$. As $m=1, \operatorname{ker}\left(C_{s}\right) \subseteq H_{s}$ and Lemma 2.6 yields $P C_{d} \subseteq C_{d} P$. In particular, we obtain that $\left(P u_{\varepsilon}\right)_{\varepsilon}$ weakly converges to $P \tilde{u}$ in $\operatorname{dom}\left(C_{d}\right)$. Furthermore, by $\mathcal{T}_{\varepsilon} v=v$ on $\operatorname{ker}\left(C_{s}\right)$ (see (14)), we infer $\mathcal{T}_{\varepsilon} P=P$. Moreover, from Lemma 2.5, we have $\mathcal{T}_{\varepsilon} P=P \mathcal{T}_{\varepsilon}$. Thus, we get that

$$
u=P \mathrm{~W}-\lim _{\varepsilon \rightarrow 0} \mathcal{T}_{\varepsilon} u_{\varepsilon}=\mathrm{w}-\lim _{\varepsilon \rightarrow 0} P \mathcal{T}_{\varepsilon} u_{\varepsilon}=\mathrm{w}-\lim _{\varepsilon \rightarrow 0} \mathcal{T}_{\varepsilon} P u_{\varepsilon}=\mathrm{w}-\lim _{\varepsilon \rightarrow 0} P u_{\varepsilon}=P \tilde{u} \in \operatorname{dom}\left(C_{d}\right) .
$$

We define $v:=\bar{v}-C_{d} u$. Then we compute for suitable $\varepsilon>0$ and all $w \in \operatorname{ker}\left(C_{s}^{*}\right) \cap \operatorname{dom}\left(C_{d}^{*}\right)$ :

$$
\left\langle\mathcal{T}_{\varepsilon} C_{d} u_{\varepsilon}, w\right\rangle=\left\langle u_{\varepsilon}, \mathcal{T}_{-\varepsilon} C_{d}^{*} w+\frac{1}{\varepsilon} \mathcal{T}_{-\varepsilon} C_{s}^{*} w\right\rangle=\left\langle\mathcal{T}_{\varepsilon} u_{\varepsilon}, C_{d}^{*} w\right\rangle
$$

Letting $\varepsilon \rightarrow 0$ on both sides, we obtain

$$
\left\langle C_{d} u+v, w\right\rangle=\left\langle C_{d} u, w\right\rangle
$$

which leads to

$$
\langle v, w\rangle=0 \quad\left(w \in \operatorname{ker}\left(C_{s}^{*}\right) \cap \operatorname{dom}\left(C_{d}^{*}\right)\right) .
$$

Since $\operatorname{ker}\left(C_{s}^{*}\right) \cap \operatorname{dom}\left(C_{d}^{*}\right)$ is dense in $\operatorname{ker}\left(C_{s}^{*}\right)$ by (10), we infer $v \in \operatorname{ker}\left(C_{s}^{*}\right)^{\perp}=\overline{\operatorname{ran}}\left(C_{s}\right)$.

Finally, we let $w \in \operatorname{ker}\left(C_{s}\right) \cap \operatorname{dom}\left(C_{d}^{*}\right)$. Then, using $\mathcal{T}_{\varepsilon} v=v$ for all $v \in \operatorname{ker}\left(C_{s}\right)$, we have for suitable $\varepsilon>0$

$$
\left\langle\mathcal{T}_{\varepsilon} C_{d} u_{\varepsilon}, w\right\rangle=\left\langle C_{d} u_{\varepsilon}, w\right\rangle=\left\langle u_{\varepsilon}, C_{d}^{*} w\right\rangle=\left\langle u_{\varepsilon}, \mathcal{T}_{-\varepsilon} C_{d}^{*} w\right\rangle=\left\langle\mathcal{T}_{\varepsilon} u_{\varepsilon}, C_{d}^{*} w\right\rangle
$$

Letting $\varepsilon \rightarrow 0$, we get

$$
\left\langle C_{d} u+v, w\right\rangle=\left\langle u, C_{d}^{*} w\right\rangle=\left\langle C_{d} u, w\right\rangle .
$$

Hence, since $\operatorname{dom}\left(C_{d}^{*}\right) \cap \operatorname{ker}\left(C_{s}\right)$ is dense in $\operatorname{ker}\left(C_{s}\right)$ as $m=1$, we deduce

$$
\langle v, w\rangle=0 \quad\left(w \in \operatorname{ker}\left(C_{s}\right)\right)
$$

which leads to $v \in \overline{\operatorname{ran}}\left(C_{s}^{*}\right)$.

\section{$3 \quad$ Homogenization of (abstract) elliptic problems}

In order to illustrate our so far findings, we shall treat an elliptic homogenization problem. Note that this is the abstract variant of the classical result [4, Theorem 4.1.1] (see Section 5.2 for the periodic case). We assume throughout that $m=1$ and that (10)-(14).

Theorem 3.1. Let $f \in \operatorname{ker}\left(C_{s}\right)$ and assume that $\operatorname{ran}\left(C_{d}\right)$ is closed and $C_{d}$ is injective. Let $A \in$ $L\left(\left(H_{d} \otimes H_{s}\right)^{n}\right)$ satisfy $\operatorname{Re} A=\frac{1}{2}\left(A+A^{*}\right) \geqslant c$ for some $c>0$. For $\varepsilon>0$ consider

$$
C_{d}^{*} \mathcal{T}_{-\varepsilon} A \mathcal{T}_{\varepsilon} C_{d} u_{\varepsilon}=f
$$


Then $\left(u_{\varepsilon}\right)_{\varepsilon}$ is well-defined and uniformly bounded in $\operatorname{dom}\left(C_{d}\right)$ and strongly 2-scale converges to some $u \in \operatorname{dom}\left(C_{d}\right) \cap \operatorname{ker}\left(C_{s}\right)$. Moreover, $\left(C_{d} u_{\varepsilon}\right)_{\varepsilon}$ weakly 2-scale converges to $C_{d} u+v$ for some $v \in \overline{\operatorname{ran}}\left(C_{s}\right) \cap \overline{\operatorname{ran}}\left(C_{s}^{*}\right)^{n}$, where $u, v$ are the unique solutions of the following system of equations

$$
\begin{aligned}
C_{d}^{*} P A\left(C_{d} u+v\right) & =f, \\
C_{s}^{*} A\left(C_{d} u+v\right) & =0 .
\end{aligned}
$$

Here, $P$ is the orthogonal projection onto $\operatorname{ker}\left(C_{s}\right)$.

Before we come to a proof of Theorem 3.1, we address well-posedness of (16). For this, we will use the direct approach outlined in [37, Theorem 3.1]; see also [49, Theorem 2.9].

Theorem 3.2. Assume the conditions of Theorem 3.1 to be in effect. Then for all $\varepsilon>0$ there exists a unique $u_{\varepsilon} \in \operatorname{dom}\left(C_{d}\right)$ satisfying (16). Moreover, we have that $\left(u_{\varepsilon}\right)_{\varepsilon}$ is uniformly bounded in $\operatorname{dom}\left(C_{d}\right)$.

Proof. Let $\iota: \operatorname{ran}\left(C_{d}\right) \hookrightarrow\left(H_{d} \otimes H_{s}\right)^{n}$ denote the canonical embedding. Then $\iota^{*}$ is the orthogonal projection onto $\operatorname{ran}\left(C_{d}\right)$, see [28, Lemma 3.2]. Moreover, from $\operatorname{ker}\left(C_{d}^{*}\right)=\operatorname{ran}\left(C_{d}\right)^{\perp}$, it is easy to see that

$$
C_{d}^{*} \mathcal{T}_{-\varepsilon} A \mathcal{T}_{\varepsilon} C_{d}=C_{d}^{*} \iota \iota^{*} \mathcal{T}_{-\varepsilon} A \mathcal{T}_{\varepsilon} \iota \iota^{*} C_{d}
$$

By the closed graph theorem $\iota^{*} C_{d}$ is continuously invertible; by [37, Lemma 2.4 and Corollary 2.5] $\left(\iota^{*} C_{d}\right)^{*}=C_{d}^{*} \iota$ is also continuously invertible. Next, from $\operatorname{Re} A \geqslant c$ it follows that $\operatorname{Re} \mathcal{T}_{-\varepsilon} A \mathcal{T}_{\varepsilon} \geqslant c$ In consequence, we obtain $\operatorname{Re} \iota^{*} \mathcal{T}_{-\varepsilon} A \mathcal{T}_{\varepsilon} \iota \geqslant c \iota^{*} \iota$. Thus, using that $\operatorname{ran}\left(C_{d}^{*}\right)$ is closed and dense in $H_{d} \otimes H_{s}$ by the injectivity of $C_{d}$, we obtain

$$
u_{\varepsilon}=\left(\iota^{*} C_{d}\right)^{-1}\left(\iota^{*} \mathcal{T}_{-\varepsilon} A \mathcal{T}_{\varepsilon} \iota\right)^{-1}\left(C_{d}^{*} \iota\right)^{-1} f,
$$

which yields uniqueness of solutions of (16). Multiplying this equality by $\iota^{*} C_{d}$, we infer

$$
\iota^{*} C_{d} u_{\varepsilon}=\left(\iota^{*} \mathcal{T}_{-\varepsilon} A \mathcal{T}_{\varepsilon} \iota\right)^{-1}\left(C_{d}^{*} \iota\right)^{-1} f .
$$

The equalities (19) and (20) together with

$$
\left\|\left(\iota^{*} \mathcal{T}_{-\varepsilon} A \mathcal{T}_{\varepsilon} \iota\right)^{-1}\right\| \leqslant \frac{1}{c}
$$

yield that $\left(u_{\varepsilon}\right)_{\varepsilon}$ is uniformly bounded in $\operatorname{dom}\left(C_{d}\right)$.

The next result settles uniqueness of the homogenized equations stated in Theorem 3.1. The rationale is similar to the one in [4, Theorem 4.1.1]; however we do not need to impose the curlcondition nor do we use any variant of 'Kozlov's identity' (see [4, Lemma 2.4 and the subsequent remark]).

Lemma 3.3. Let $u \in \operatorname{dom}\left(C_{d}\right) \cap \operatorname{ker}\left(C_{s}\right)$ and $v \in \overline{\operatorname{ran}}\left(C_{s}\right) \cap \overline{\operatorname{ran}}\left(C_{s}^{*}\right)^{n}$ satisfy (17) and (18) with $f=0$. Assume that $C_{d}$ is injective. Then $u=0$ and $v=0$.

Proof. We define $\zeta:=C_{d} u+v$. Since $\zeta-C_{d} u=v \in \overline{\operatorname{ran}}\left(C_{s}\right)$ and $A\left(C_{d} u+v\right) \in \operatorname{ker}\left(C_{s}^{*}\right)$, by (18),

$$
0=\langle A \zeta, v\rangle=\left\langle A \zeta, \zeta-C_{d} u\right\rangle .
$$

Thus, we deduce from (17) with $f=0$ using $u \in \operatorname{dom}\left(C_{d}\right) \cap \operatorname{ker}\left(C_{s}\right)$

$$
\langle A \zeta, \zeta\rangle=\left\langle A \zeta, C_{d} u\right\rangle=\left\langle A \zeta, C_{d} P u\right\rangle=\left\langle P A \zeta, C_{d} u\right\rangle=\left\langle C_{d}^{*} P A \zeta, u\right\rangle=0 .
$$

Thus, by $\operatorname{Re} A \geqslant c$, we infer $\zeta=0$. Since $\operatorname{ker}\left(C_{s}\right)^{n} \ni C_{d} u \perp v \in \overline{\operatorname{ran}}\left(C_{s}^{*}\right)^{n}$, we obtain the assertion. 
The following simple lemma will be useful in the proof of Theorem 3.1. It provides a recovery construction for the weak two-scale limit of the sequence $C_{d} u_{\varepsilon}$.

Lemma 3.4. Let $u \in \operatorname{dom}\left(C_{d}\right) \cap \operatorname{ker}\left(C_{s}\right)$ and $v \in \overline{\operatorname{ran}}\left(C_{s}\right)$. For $\delta>0$, there exists $\varphi_{\delta} \in \operatorname{dom}\left(C_{d}\right) \cap$ $\operatorname{dom}\left(C_{s}\right)$ such that

$$
\left\|C_{s} \varphi_{\delta}-v\right\| \leqslant \delta .
$$

Moreover, for $\varphi_{\delta, \varepsilon}:=\mathcal{T}_{-\varepsilon} u+\varepsilon \mathcal{T}_{-\varepsilon} \varphi_{\delta}$, we obtain

$$
\mathcal{T}_{\varepsilon} \varphi_{\delta, \varepsilon} \rightarrow u, \quad \mathcal{T}_{\varepsilon} C_{d} \varphi_{\delta, \varepsilon} \rightarrow C_{d} u+C_{s} \varphi_{\delta} \quad(\text { as } \varepsilon \rightarrow 0)
$$

Proof. By the definition of $\overline{\operatorname{ran}}\left(C_{s}\right)$ and using (10), we obtain that there exists $\varphi_{\delta} \in \operatorname{dom}\left(C_{d}\right) \cap$ $\operatorname{dom}\left(C_{s}\right)$ which satisfies (21). Also, since $\mathcal{T}_{\varepsilon} \varphi_{\delta, \varepsilon}=u+\varepsilon \varphi_{\delta}$, it follows that $\mathcal{T}_{\varepsilon} \varphi_{\delta, \varepsilon} \rightarrow u$. Furthermore, using (12)-(13) we compute

$$
C_{d} \varphi_{\delta, \varepsilon}=\mathcal{T}_{-\varepsilon} C_{d} u+\varepsilon \mathcal{T}_{-\varepsilon} C_{d} \varphi_{\delta}+\mathcal{T}_{-\varepsilon} C_{s} \varphi_{\delta}
$$

This implies that $\mathcal{T}_{\varepsilon} C_{d} \varphi_{\delta, \varepsilon} \rightarrow C_{d} u+C_{s} \varphi_{\delta}$.

Proof of Theorem 3.1. The family $\left(u_{\varepsilon}\right)_{\varepsilon>0}$ is well-defined and bounded in $\operatorname{dom}\left(C_{d}\right)$ by Theorem 3.2. By Theorem 2.8 (c), we find a subsequence of $\left(u_{\varepsilon}\right)_{\varepsilon>0}, u \in \operatorname{dom}\left(C_{d}\right) \cap \operatorname{ker}\left(C_{s}\right), v \in \overline{\operatorname{ran}}\left(C_{s}^{*}\right)^{n} \cap \overline{\operatorname{ran}}\left(C_{s}\right)$ such that $\left(u_{\varepsilon}\right)_{\varepsilon}$ and $\left(C_{d} u_{\varepsilon}\right)_{\varepsilon}$ weakly 2 -scale converge to $u$ and $C_{d} u+v$, respectively. Let $\varphi \in \operatorname{dom}\left(C_{d}\right)$ and $\psi \in \operatorname{dom}\left(C_{s}\right) \cap \operatorname{dom}\left(C_{d}\right)$. Then we compute for suitable $\varepsilon>0$ using (16)

$$
\begin{aligned}
\langle f, \varphi\rangle+\left\langle\varepsilon f, \mathcal{T}_{-\varepsilon} \psi\right\rangle & =\left\langle\mathcal{T}_{-\varepsilon} A \mathcal{T}_{\varepsilon} C_{d} u_{\varepsilon}, C_{d} P \varphi+\varepsilon C_{d} \mathcal{T}_{-\varepsilon} \psi\right\rangle \\
& =\left\langle\mathcal{T}_{-\varepsilon} A \mathcal{T}_{\varepsilon} C_{d} u_{\varepsilon}, C_{d} P \varphi+\varepsilon \mathcal{T}_{-\varepsilon} C_{d} \psi+C_{s} \mathcal{T}_{-\varepsilon} \psi\right\rangle \\
& =\left\langle A \mathcal{T}_{\varepsilon} C_{d} u_{\varepsilon}, \mathcal{T}_{\varepsilon} C_{d} P \varphi\right\rangle+\left\langle A \mathcal{T}_{\varepsilon} C_{d} u_{\varepsilon}, \varepsilon C_{d} \psi\right\rangle+\left\langle A \mathcal{T}_{\varepsilon} C_{d} u_{\varepsilon}, C_{s} \psi\right\rangle
\end{aligned}
$$

where we used $\varepsilon C_{d} \mathcal{T}_{-\varepsilon} \psi=\varepsilon \mathcal{T}_{-\varepsilon} C_{d} \psi+C_{s} \mathcal{T}_{-\varepsilon} \psi$ and $\mathcal{T}_{-\varepsilon} C_{s} \psi=C_{s} \mathcal{T}_{-\varepsilon} \psi$. Since $C_{d} P \varphi \in \operatorname{ker}\left(C_{s}\right)$, we have $\mathcal{T}_{\varepsilon} C_{d} P \varphi=\mathcal{T}_{\varepsilon} P C_{d} \varphi=P C_{d} \varphi$. Thus, we obtain

$$
\langle f, \varphi\rangle+\left\langle\varepsilon f, \mathcal{T}_{-\varepsilon} \psi\right\rangle=\left\langle A \mathcal{T}_{\varepsilon} C_{d} u_{\varepsilon}, P C_{d} \varphi\right\rangle+\left\langle A \mathcal{T}_{\varepsilon} C_{d} u_{\varepsilon}, \varepsilon C_{d} \psi\right\rangle+\left\langle A \mathcal{T}_{\varepsilon} C_{d} u_{\varepsilon}, C_{s} \psi\right\rangle
$$

In this equality we may let $\varepsilon \rightarrow 0$ and obtain the asserted equalities (17) and (18). As the solutions to (17) and (18) are unique by Lemma 3.3, we deduce that any subsequence of $\left(u_{\varepsilon}\right)_{\varepsilon}$ and $\left(C_{d} u_{\varepsilon}\right)_{\varepsilon}$ weakly 2 -scale converges to $u$ and $C_{d} u+v$, which implies that both $\left(u_{\varepsilon}\right)_{\varepsilon}$ and $\left(C_{d} u_{\varepsilon}\right)_{\varepsilon}$ weakly 2-scale converge without the choice of subsequences.

In order to show strong convergence of $\left(\mathcal{T}_{\varepsilon} u_{\varepsilon}\right)_{\varepsilon}$, we consider $\varphi_{\delta}$ and $\varphi_{\delta, \varepsilon}$ defined as in Lemma 3.4 (for $u, v$ the solution of (17)-(18)). Since $C_{d}$ is injective and it has closed range, it follows (see, e.g., $\left[37\right.$, Remark 3.2(b)]) that there exists $c_{0}>0$ such that $\|\varphi\| \leqslant c_{0}\left\|C_{d} \varphi\right\|$ for all $\varphi \in \operatorname{dom}\left(C_{d}\right)$. As a result of this, we obtain

$$
\left\|u_{\varepsilon}-\varphi_{\delta, \varepsilon}\right\| \leqslant c_{0}\left\|C_{d}\left(u_{\varepsilon}-\varphi_{\delta, \varepsilon}\right)\right\|=c_{0}\left\|\mathcal{T}_{\varepsilon} C_{d}\left(u_{\varepsilon}-\varphi_{\delta, \varepsilon}\right)\right\| .
$$

Using the assumption $\operatorname{Re} A \geqslant c$ (in the inequality) and that $u_{\varepsilon}$ solves (16) (in the equality), we obtain

$$
\begin{aligned}
c\left\|\mathcal{T}_{\varepsilon} C_{d}\left(u_{\varepsilon}-\varphi_{\delta, \varepsilon}\right)\right\|^{2} & \leqslant \operatorname{Re}\left\langle A \mathcal{T}_{\varepsilon} C_{d}\left(u_{\varepsilon}-\varphi_{\delta, \varepsilon}\right), \mathcal{T}_{\varepsilon} C_{d}\left(u_{\varepsilon}-\varphi_{\delta, \varepsilon}\right)\right\rangle \\
& =\operatorname{Re}\left\langle f, u_{\varepsilon}-\varphi_{\delta, \varepsilon}\right\rangle-\operatorname{Re}\left\langle A \mathcal{T}_{\varepsilon} C_{d} \varphi_{\delta, \varepsilon}, \mathcal{T}_{\varepsilon} C_{d}\left(u_{\varepsilon}-\varphi_{\delta, \varepsilon}\right)\right\rangle .
\end{aligned}
$$


Since $f \in \operatorname{ker}\left(\mathcal{T}_{\varepsilon}-1\right.$ ) (for any $\varepsilon \neq 0$ ), we conclude that the first term on the right-hand side equals $\operatorname{Re}\left\langle f, \mathcal{T}_{\varepsilon} u_{\varepsilon}-\mathcal{T}_{\varepsilon} \varphi_{\delta, \varepsilon}\right\rangle$. As a result of this and using the properties of $\varphi_{\delta, \varepsilon}$ (Lemma 3.4), we obtain that for fixed $\delta>0$,

$$
\limsup _{\varepsilon \rightarrow 0}\left\|\mathcal{T}_{\varepsilon} C_{d}\left(u_{\varepsilon}-\varphi_{\delta, \varepsilon}\right)\right\|^{2} \leqslant-\operatorname{Re}\left\langle A\left(C_{d} u+C_{s} \varphi_{\delta}\right), v-C_{s} \varphi_{\delta}\right\rangle .
$$

Moreover, using the above and (22) we obtain (using that the second term vanishes in the limit $\varepsilon \rightarrow 0$ by Lemma 3.4) for some $c_{1} \geqslant 0$ and all $\delta>0$

$$
\limsup _{\varepsilon \rightarrow 0}\left(\left\|u_{\varepsilon}-\varphi_{\delta, \varepsilon}\right\|^{2}+\left\|\mathcal{T}_{\varepsilon} \varphi_{\delta, \varepsilon}-u\right\|^{2}+\left\|\mathcal{T}_{\varepsilon} C_{d}\left(u_{\varepsilon}-\varphi_{\delta, \varepsilon}\right)\right\|^{2}\right) \leqslant c_{1}\left\|C_{d} u+C_{s} \varphi_{\delta}\right\|\left\|v-C_{s} \varphi_{\delta}\right\| .
$$

By the choice of $\varphi_{\delta}$ (Lemma 3.4), in the limit $\delta \rightarrow 0$ the right-hand side vanishes. Consequently, we find a diagonal sequence $\delta(\varepsilon) \rightarrow 0$ (as $\varepsilon \rightarrow 0$ ) such that

$$
\left\|u_{\varepsilon}-\varphi_{\delta(\varepsilon), \varepsilon}\right\|+\left\|\mathcal{T}_{\varepsilon} \varphi_{\delta(\varepsilon), \varepsilon}-u\right\|+\left\|\mathcal{T}_{\varepsilon} C_{d}\left(u_{\varepsilon}-\varphi_{\delta(\varepsilon), \varepsilon}\right)\right\| \rightarrow 0 \quad \text { as } \varepsilon \rightarrow 0
$$

Finally, this yields

$$
\left\|\mathcal{T}_{\varepsilon} u_{\varepsilon}-u\right\| \leqslant\left\|\mathcal{T}_{\varepsilon} u_{\varepsilon}-\mathcal{T}_{\varepsilon} \varphi_{\delta(\varepsilon), \varepsilon}\right\|+\left\|\mathcal{T}_{\varepsilon} \varphi_{\delta(\varepsilon), \varepsilon}-u\right\| \rightarrow 0 \quad \text { as } \varepsilon \rightarrow 0 .
$$

The above proof implies the following abstract corrector type result (see (23)).

Corollary 3.5. Assume the conditions of Theorem 3.1 to be in effect and let $u_{\varepsilon}, u, v$ be given as in Theorem 3.1. There exists a sequence $\varphi_{\varepsilon} \in \operatorname{dom}\left(C_{d}\right) \cap \operatorname{dom}\left(C_{s}\right)$ such that

$$
\varepsilon \varphi_{\varepsilon} \rightarrow 0, \quad \mathcal{T}_{\varepsilon} C_{d}\left(u_{\varepsilon}-\varepsilon \mathcal{T}_{-\varepsilon} \varphi_{\varepsilon}\right) \rightarrow C_{d} u \text { strongly in } H_{d} \otimes H_{s} .
$$

\section{Homogenization of abstract evolutionary equations}

\subsection{A Hilbert space framework for evolutionary equations}

For the application to time-dependent problems in the subsequent sections, we shall specify the particular framework, we are working in. For this, we recall some results from [26], where the setting was introduced for the first time. We shall also refer to [16, Section 2] for more details, when properties of the time-derivative to be introduced in the following are concerned.

To begin with, we define for $\nu \in \mathbb{R}_{\geqslant 0}$ the space $L_{\nu}^{2}(\mathbb{R} ; H)$ of (equivalence classes of) Hilbert space $H$-valued functions $f$ being Bochner measurable and satisfy

$$
\|f\|_{\nu}^{2}:=\int_{\mathbb{R}}\|f(t)\|_{H}^{2} \exp (-2 \nu t) d t<\infty .
$$

$L_{\nu}^{2}(\mathbb{R} ; H)$ is a Hilbert space under the norm $\|\cdot\|_{\nu}$. Denoting by $H_{\nu}^{1}(\mathbb{R} ; H)$ the (first) Sobolev space of weakly differentiable functions with distributional derivative representable as an element in $L_{\nu}^{2}(\mathbb{R} ; H)$, we define the time-derivative as the operator

$$
\partial_{t, \nu}: H_{\nu}^{1}(\mathbb{R} ; H) \subseteq L_{\nu}^{2}(\mathbb{R} ; H) \rightarrow L_{\nu}^{2}(\mathbb{R} ; H), f \mapsto f^{\prime} .
$$

It turns out that $\partial_{t, \nu}$ is a normal operator. More particularly, $\partial_{t, \nu}$ admits a spectral representation as multiplication operator in $L^{2}(\mathbb{R} ; H)$. For this, we define the multiplication-by-the-argument operator

$$
\mathrm{m}: \operatorname{dom}(\mathrm{m}) \subseteq L^{2}(\mathbb{R} ; H) \rightarrow L^{2}(\mathbb{R} ; H), \varphi \mapsto(\xi \mapsto \xi \varphi(\xi)),
$$


where

$$
\operatorname{dom}(\mathrm{m}):=\left\{\varphi \in L^{2}(\mathbb{R} ; H) ;(\xi \mapsto \xi \varphi(\xi)) \in L^{2}(\mathbb{R} ; H)\right\} .
$$

Define the $\left(H\right.$-valued) Fourier transformation $\mathcal{F}: L^{2}(\mathbb{R} ; H) \rightarrow L^{2}(\mathbb{R} ; H)$ as the unitary extension of

$$
\mathcal{F} f:=\left(\xi \mapsto \frac{1}{\sqrt{2 \pi}} \int_{\mathbb{R}} \exp (-1 t \xi) f(t) d t\right) \quad\left(f \in L^{1}(\mathbb{R} ; H) \cap L^{2}(\mathbb{R} ; H)\right) .
$$

Moreover, we define for $\nu \in \mathbb{R}_{\geqslant 0}$ the (obviously) unitary operator

$$
\exp (-\nu \mathrm{m}): L_{\nu}^{2}(\mathbb{R} ; H) \rightarrow L^{2}(\mathbb{R} ; H), f \mapsto\left(t \mapsto \mathrm{e}^{-\nu t} f(t)\right),
$$

and we set

$$
\mathcal{L}_{\nu}:=\mathcal{F} \exp (-\nu \mathrm{m})
$$

the Fourier-Laplace transformation. With the latter transformation at hand the explicit spectral theorem for $\partial_{t, \nu}$ (see also [16, Corollary 2.5(c)]) reads

$$
\partial_{t, \nu}=\mathcal{L}_{\nu}^{*}(\mathrm{~lm}+\nu) \mathcal{L}_{\nu}
$$

The latter equation yields a functional calculus for $\partial_{t, \nu}$ (or for $\partial_{t, \nu}^{-1}$.). In fact, let $\nu_{0} \in \mathbb{R}_{\geqslant 0}$ and $\nu>\nu_{0}$ and let $\mathcal{M} \in \mathcal{H}\left(\mathbb{C}_{\operatorname{Re}>\nu_{0}} ; L(H, K)\right)$, where $K$ is another Hilbert space and

$$
\mathcal{H}\left(\mathbb{C}_{\operatorname{Re}>\nu_{0}} ; L(H, K)\right):=\left\{\mathcal{M}: \mathbb{C}_{\operatorname{Re}>\nu_{0}} \rightarrow L(H, K) ; \mathcal{M} \text { analytic }\right\} .
$$

Then we define

$$
\mathcal{M}\left(\partial_{t, \nu}\right):=\mathcal{L}_{\nu}^{*}(\mathcal{M}(\mathrm{m}+\nu)) \mathcal{L}_{\nu}
$$

where

$$
(\mathcal{M}(1 \mathrm{~m}+\nu) \varphi)(\xi):=\mathcal{M}\left({ }_{1} \xi+\nu\right) \varphi(\xi) \quad(\xi \in \mathbb{R})
$$

for all compactly supported, continuous functions $\varphi: \mathbb{R} \rightarrow H$.

The reason we focus on analytic functions $\mathcal{M}$ rather than continuous or even just measurable functions is that analyticity of $\mathcal{M}$ and causality of $\mathcal{M}\left(\partial_{t, \nu}\right)$ are strongly related, which is apparent from the Paley-Wiener theorem, see e.g., [26, Section 2] or [29, Theorem 2.4], [46, Section 1.2].

We are now in the position to formulate the well-posedness theorem, which can be viewed as underlying structure of many linear equations in mathematical physics and continuum mechanics.

Theorem 4.1 ([26, Solution Theory $])$. Let $\nu_{0} \in \mathbb{R}_{\geqslant 0}, \nu>\nu_{0}, H$ Hilbert space, $A: \operatorname{dom}(A) \subseteq H \rightarrow$ $H$ a skew-self-adjoint operator, i.e., $A=-A^{*}$, and $\mathcal{M} \in \mathcal{H}\left(\mathbb{C}_{\operatorname{Re}>\nu_{0}} ; L(H)\right)$. Assume there exists $c>0$ such that

$$
\operatorname{Re}\langle\mathcal{M}(z) \varphi, \varphi\rangle \geqslant c\langle\varphi, \varphi\rangle \quad\left(z \in \mathbb{C}_{\operatorname{Re}>\nu_{0}}, \varphi \in H\right) .
$$

Then the operator $B:=\mathcal{M}\left(\partial_{t, \nu}\right)+A$ is densely defined on $L_{\nu}^{2}(\mathbb{R} ; H)$. Moreover, $B$ is closable and $\bar{B}$ is continuously invertible in $L_{\nu}^{2}(\mathbb{R} ; H),\left\|\bar{B}^{-1}\right\| \leqslant 1 / c$ and $\bar{B}^{-1}$ is causal, that is,

$$
\mathbf{1}_{\mathbb{R}_{\leqslant a}} \bar{B}^{-1} \mathbf{1}_{\mathbb{R}_{\leqslant a}}=\mathbf{1}_{\mathbb{R}_{\leqslant a}} \bar{B}^{-1} \quad(a \in \mathbb{R}) .
$$

Proof. For $z \in \mathbb{C}_{\operatorname{Re}>\nu_{0}}$ it follows from [34, Lemma 2.12] that $\mathcal{M}(z)+A$ is continuously invertible in $H$ with inverse satisfying $\left\|(\mathcal{M}(z)+A)^{-1}\right\| \leqslant 1 / c$. Thus, [36, Remark $2.3($ a) $]$ applies and we obtain the assertion. 
We define

$$
\mathcal{H}^{\infty}\left(\mathbb{C}_{\operatorname{Re}>\nu_{0}} ; L(H, K)\right):=\left\{\mathcal{M} \in \mathcal{H}\left(\mathbb{C}_{\operatorname{Re}>\nu_{0}} ; L(H, K)\right) ; \mathcal{M} \text { bounded }\right\}
$$

which turns into a Banach space if endowed with the supremum norm.

Proposition 4.2. Assume the hypotheses of Theorem 4.1. Then

$$
\left[\mathbb{C}_{\operatorname{Re}>\nu_{0}} \ni z \mapsto(\mathcal{M}(z)+A)^{-1} \in L(H)\right] \in \mathcal{H}^{\infty}\left(\mathbb{C}_{\operatorname{Re}>\nu} ; L(H)\right) .
$$

Proof. The claim follows from [34, Lemma 2.12] and the fact that composition of analytic mappings are analytic again.

It will be the next theorem, which forms the basic result for the convergence results to be followed in the next section.

Theorem 4.3. Let $\nu_{0} \in \mathbb{R}_{\geqslant 0}, H, K$ separable Hilbert spaces. Let $\left(S_{\varepsilon}\right)_{\varepsilon>0}$ be a bounded family in $\mathcal{H}^{\infty}\left(\mathbb{C}_{\operatorname{Re}>\nu_{0}} ; L(H, K)\right)$ and let $S_{0} \in \mathcal{H}^{\infty}\left(\mathbb{C}_{\operatorname{Re}>\nu_{0}} ; L(H, K)\right)$. Assume that for all $z \in \mathbb{C}_{\operatorname{Re}>\nu_{0}}$ we have

$$
S_{\varepsilon}(z) \rightarrow S_{0}(z) \quad(\varepsilon \rightarrow 0)
$$

in the weak operator topology of $L(H, K)$.

Then $S_{\varepsilon}\left(\partial_{t, \nu}\right) \rightarrow S_{0}\left(\partial_{t, \nu}\right)$ in the weak operator topology of $L\left(L_{\nu}^{2}(\mathbb{R} ; H), L_{\nu}^{2}(\mathbb{R} ; K)\right)$.

For the proof of Theorem 4.3, we shall use the following result.

Theorem 4.4 ([44, Theorem 4.3] and [41, Lemma 3.5]). Let $\Omega \subseteq \mathbb{C}$ open, $H, K$ separable Hilbert spaces. Let $\left(S_{\varepsilon}\right)_{\varepsilon}$ be a bounded family in $\mathcal{H}^{\infty}\left(\mathbb{C}_{\mathrm{Re}>\nu_{0}} ; L(H, K)\right)$. Then there exists

$$
T \in \mathcal{H}^{\infty}\left(\mathbb{C}_{\operatorname{Re}>\nu_{0}} ; L(H, K)\right)
$$

and a nullsequence $\left(\varepsilon_{k}\right)_{k \in \mathbb{N}}$ in $(0, \infty)$ satisfying for all $K \subseteq \Omega$ compact

$$
S_{\varepsilon_{k}}(z) \rightarrow T(z) \quad(k \rightarrow \infty, z \in K)
$$

in the weak operator topology of $L(H, K)$. Moreover, if $\Omega=\mathbb{C}_{\mathrm{Re}>\nu_{0}}$, then

$$
S_{\varepsilon_{k}}\left(\partial_{t, \nu}\right) \rightarrow T\left(\partial_{t, \nu}\right) \quad(k \rightarrow \infty)
$$

in the weak operator topology of $L\left(L_{\nu}^{2}(\mathbb{R} ; H), L_{\nu}^{2}(\mathbb{R} ; K)\right)$ for all $\nu>\nu_{0}$.

Proof of Theorem 4.3. Choose $\left(\varepsilon_{k}\right)_{k}$ and $T$ according to Theorem 4.4. Then, for $z \in \mathbb{C}_{\operatorname{Re}>\nu_{0}}$, we obtain

$$
S_{0}(z)=\left(\tau_{\mathrm{w}^{-}}\right) \lim _{k \rightarrow \infty} S_{\varepsilon_{k}}(z)=T(z)
$$

Moreover, we get $S_{\varepsilon_{k}}\left(\partial_{t, \nu}\right) \rightarrow S_{0}\left(\partial_{t, \nu}\right)$ in the weak operator topology of $L\left(L_{\nu}^{2}(\mathbb{R} ; H), L_{\nu}^{2}(\mathbb{R} ; K)\right)$. The subsequence principle concludes the proof. 


\subsection{Applications to time-dependent-type problems}

We shall treat a subclass of evolutionary equations discussed in the previous section. The particular cases treated here cover the heat equation, the wave equation, the Maxwell's equations and even systems of mixed type formulated on possibly rough domains, which do not need to satisfy any boundedness conditions, as we shall demonstrate in the subsequent sections by means of examples.

More specifically, in this section, we confine ourselves to the following class of problems. Define $H_{0}:=\left(H_{d} \otimes H_{s}\right)^{m}, H_{1}:=\left(H_{d} \otimes H_{s}\right)^{n}$ and $H=H_{0} \oplus H_{1}$. Let $\nu_{0} \geqslant 0, \mathcal{M}_{k}: \mathbb{C}_{\operatorname{Re}>\nu_{0}} \rightarrow L\left(H_{k}\right)$ be analytic and assume that $\operatorname{Re} \mathcal{M}_{k}(z) \geqslant c$ for all $z \in \mathbb{C}_{\operatorname{Re}>\nu_{0}}, k \in\{0,1\}$ and some $c>0$. We need to restrict ourselves to a certain class of right-hand sides. For this we set

$$
\mathcal{H}_{0}:=\operatorname{ker}\left(C_{s}\right) \cap \bigcap_{\varepsilon \in \mathbb{R} \backslash\{0\}} \operatorname{ker}\left(\mathcal{T}_{\varepsilon}-1\right) \subseteq H_{0}, \quad \mathcal{H}_{1}:=\operatorname{ker}\left(C_{s}^{*}\right) \cap \bigcap_{\varepsilon \in \mathbb{R} \backslash\{0\}} \operatorname{ker}\left(\mathcal{T}_{\varepsilon}-1\right) \subseteq H_{1}
$$

We remark that in the applications, e.g., to stochastic homogenization (see Sections 5.3 and 5.4), the above choice allows the consideration of deterministic right-hand sides (that is a standard assumption in stochastic homogenization), i.e., $L^{2}(Q) \otimes \mathbb{C} \subseteq \mathcal{H}_{i}$.

For $(f, g) \in \mathcal{H}:=\mathcal{H}_{0} \oplus \mathcal{H}_{1}$, we consider for $\varepsilon>0$

$$
\left(\mathcal{T}_{-\varepsilon}\left(\begin{array}{cc}
\mathcal{M}_{0}(z) & 0 \\
0 & \mathcal{M}_{1}(z)
\end{array}\right) \mathcal{T}_{\varepsilon}+\left(\begin{array}{cc}
0 & C_{d}^{*} \\
-C_{d} & 0
\end{array}\right)\right)\left(\begin{array}{l}
u_{\varepsilon} \\
q_{\varepsilon}
\end{array}\right)=\left(\begin{array}{l}
f \\
g
\end{array}\right)
$$

Note that the operator

$$
B_{\varepsilon}:=\overline{\left(\mathcal{T}_{-\varepsilon}\left(\begin{array}{cc}
\mathcal{M}_{0}\left(\partial_{t, \nu}\right) & 0 \\
0 & \mathcal{M}_{1}\left(\partial_{t, \nu}\right)
\end{array}\right) \mathcal{T}_{\varepsilon}+\left(\begin{array}{cc}
0 & C_{d}^{*} \\
-C_{d} & 0
\end{array}\right)\right)}
$$

is continuously invertible in $L_{\nu}^{2}(\mathbb{R} ; H)$ by Theorem 4.1 applied to $\mathcal{M}=\mathcal{T}_{-\varepsilon} \operatorname{diag}\left(\mathcal{M}_{0}, \mathcal{M}_{1}\right) \mathcal{T}_{\varepsilon}$ and $A=\left(\begin{array}{cc}0 & C_{d}^{*} \\ -C_{d} & 0\end{array}\right)$. We shall also introduce

$$
S_{\varepsilon}(z):={\overline{\left(\mathcal{T}_{-\varepsilon}\left(\begin{array}{cc}
\mathcal{M}_{0}(z) & 0 \\
0 & \mathcal{M}_{1}(z)
\end{array}\right) \mathcal{T}_{\varepsilon}+\left(\begin{array}{cc}
0 & C_{d}^{*} \\
-C_{d} & 0
\end{array}\right)\right)}}^{-1}
$$

for all $\varepsilon>0$ and $z \in \mathbb{C}_{\operatorname{Re}>\nu_{0}}$. By Proposition 4.2, we have that $\left(S_{\varepsilon}\right)_{\varepsilon>0}$ is a bounded family in $\mathcal{H}^{\infty}\left(\mathbb{C}_{\operatorname{Re}>\nu_{0}} ; L(H)\right)$.

Our aim in this section will be to construct an operator-valued function $S_{0}: z \mapsto S_{0}(z)$ such that

$$
\mathcal{T}_{\varepsilon} S_{\varepsilon}(z) \rightarrow S_{0}(z) \quad(\varepsilon \rightarrow 0)
$$

in the weak operator topology (in an appropriate space). It will turn out that $S_{0}(z)$ can be written as $S_{0}(z)=\left(\tilde{M}(z)+\left(\begin{array}{cc}0 & C_{d}^{*} \\ -C_{d} & 0\end{array}\right)\right)^{-1}$ for suitable $\tilde{M}(z)$ to be described explicitly below. Finally, we shall conclude with an application of Theorem 4.3 to obtain a homogenization result for the full time-dependent problem.

We will suppress the dependence of $u_{\varepsilon}$ and $q_{\varepsilon}$ on $z$ for the time being; at the end of this section we come back to this. 
Remark 4.5. We refer to $[27,29,46]$ and the references therein for an instance of the many examples that are covered by this equation. We will consider some special cases in the next section. The rather involved homogenization result for a suitable class of non-diagonal $M$ is treated in [47]. In that paper, however, a compactness assumption had to be introduced, which we do not assume here. Moreover, in [47] the local problem is given implicitly and there is no criterion ensuring convergence without the extraction of subsequences. However, in the framework of socalled 'nonlocal $H$-convergence' a convergence result for Maxwell's equations was shown in [49]. For a setting strictly confined to periodic problems defined on the whole Euclidean space as underlying domain, quantitative results can be found in [11].

First of all we establish existence and boundedness of $\left(u_{\varepsilon}, q_{\varepsilon}\right)_{\varepsilon}$ in $\operatorname{dom}\left(C_{d}\right) \oplus \operatorname{dom}\left(C_{d}^{*}\right)$. The result is as plain as it is to establish the uniform bound in $H$. We provide some more details as follows.

Proposition 4.6. For all $\varepsilon>0,\left(u_{\varepsilon}, q_{\varepsilon}\right)$ is well-defined as a solution of (24). Moreover, the family $\left(u_{\varepsilon}, q_{\varepsilon}\right)_{\varepsilon}$ is uniformly bounded in $\operatorname{dom}\left(C_{d}\right) \oplus \operatorname{dom}\left(C_{d}^{*}\right)$.

Proof. Note that $\left(\begin{array}{cc}0 & C_{d}^{*} \\ -C_{d} & 0\end{array}\right)$ is skew-self-adjoint and $\operatorname{Re} \mathcal{T}_{-\varepsilon} \mathcal{M}(z) \mathcal{T}_{\varepsilon} \geqslant c$ for all $z \in \mathbb{C}_{\operatorname{Re}>\nu}, \varepsilon>0$ and some $c>0$. Hence, the assertion follows from [34, Lemma 2.12] applied to $M=\mathcal{T}_{-\varepsilon} \mathcal{M}(z) \mathcal{T}_{\varepsilon}$ and $A=\left(\begin{array}{cc}0 & C_{d}^{*} \\ -C_{d} & 0\end{array}\right)$.

The main result of this section is presented next, that is, we will now present the main step to establish convergence of $\left(\mathcal{T}_{\varepsilon} S_{\varepsilon}(z)\right)_{\varepsilon}$. We shall show convergence of a subsequence first. Then, we will prove uniqueness of the limit, so that the following theorem actually also holds without the choice of subsequences. Below, $P_{\operatorname{ker}\left(C_{s}\right)}$ and $P_{\operatorname{ker}\left(C_{s}^{*}\right)}$ denote the orthogonal projections to $\operatorname{ker}\left(C_{s}\right)$ and $\operatorname{ker}\left(C_{s}^{*}\right)$.

Theorem 4.7. Assume (10)-(13). For $\varepsilon>0$ let $\left(u_{\varepsilon}, q_{\varepsilon}\right)_{\varepsilon}$ be given by (24). Then (a subsequence of) $\left(u_{\varepsilon}\right)_{\varepsilon},\left(q_{\varepsilon}\right)_{\varepsilon}$ weakly 2-scale converge to some $u \in \operatorname{dom}\left(C_{d}\right) \cap \operatorname{ker}\left(C_{s}\right), q \in \operatorname{dom}\left(C_{d}^{*}\right) \cap \operatorname{ker}\left(C_{s}^{*}\right)$. $(u, q)$ satisfies the following system of equations

$$
\begin{aligned}
& P_{\operatorname{ker}\left(C_{s}\right)} \mathcal{M}_{0}(z) u+P_{\operatorname{ker}\left(C_{s}\right)} C_{d}^{*} q=f \\
& P_{\operatorname{ker}\left(C_{s}^{*}\right)} \mathcal{M}_{1}(z) q-P_{\operatorname{ker}\left(C_{s}^{*}\right)} C_{d} u=g .
\end{aligned}
$$

Proof. By Proposition 4.6, $\left(u_{\varepsilon}\right)_{\varepsilon}$ and $\left(q_{\varepsilon}\right)_{\varepsilon}$ are bounded in $\operatorname{dom}\left(C_{d}\right)$ and $\operatorname{dom}\left(C_{d}^{*}\right)$, respectively. Hence, by Theorem 2.8 (a) and (b), we find subsequences of $\left(u_{\varepsilon}\right)_{\varepsilon}$ and $\left(q_{\varepsilon}\right)_{\varepsilon}$ as well as $u \in \operatorname{dom}\left(C_{d}\right) \cap$ $\operatorname{ker}\left(C_{s}\right), v \in \overline{\operatorname{ran}}\left(C_{s}\right), q \in \operatorname{dom}\left(C_{d}^{*}\right) \cap \operatorname{ker}\left(C_{s}^{*}\right)$ and $w \in \overline{\operatorname{ran}}\left(C_{s}^{*}\right)$ such that $\left(u_{\varepsilon}\right)_{\varepsilon},\left(q_{\varepsilon}\right)_{\varepsilon},\left(C_{d} u_{\varepsilon}\right)_{\varepsilon}$, and $\left(C_{d}^{*} q_{\varepsilon}\right)_{\varepsilon}$ weakly 2 -scale converge to $u, q, C_{d} u+v$, and $C_{d}^{*} q+w$, respectively. Next, using (24), we have for all $\varphi \in\left(H_{d} \otimes H_{s}\right)^{m}$ and suitable $\varepsilon>0$

$$
\begin{aligned}
& \langle f, \varphi\rangle=\left\langle\mathcal{T}_{\varepsilon} f, \varphi\right\rangle \\
& =\left\langle P_{\operatorname{ker}\left(C_{s}\right)} f, \mathcal{T}_{-\varepsilon} \varphi\right\rangle \\
& =\left\langle\mathcal{T}_{-\varepsilon} \mathcal{M}_{0}(z) \mathcal{T}_{\varepsilon} u_{\varepsilon}+C_{d}^{*} q_{\varepsilon}, \mathcal{T}_{-\varepsilon} P_{\operatorname{ker}\left(C_{s}\right)} \varphi\right\rangle \\
& =\left\langle\mathcal{M}_{0}(z) \mathcal{T}_{\varepsilon} u_{\varepsilon}, P_{\operatorname{ker}\left(C_{s}\right)} \varphi\right\rangle+\left\langle\mathcal{T}_{\varepsilon} C_{d}^{*} q_{\varepsilon}, P_{\operatorname{ker}\left(C_{s}\right)} \varphi\right\rangle \\
& \rightarrow\left\langle\mathcal{M}_{0}(z) u, P_{\text {ker }\left(C_{s}\right)} \varphi\right\rangle+\left\langle C_{d}^{*} q+w, P_{\operatorname{ker}\left(C_{s}\right)} \varphi\right\rangle \\
& =\left\langle P_{\operatorname{ker}\left(C_{s}\right)} \mathcal{M}_{0}(z) u, \varphi\right\rangle+\left\langle P_{\operatorname{ker}\left(C_{s}\right)} C_{d}^{*} q, \varphi\right\rangle \quad(\varepsilon \rightarrow 0) \text {. }
\end{aligned}
$$


We obtain

$$
P_{\text {ker }\left(C_{s}\right)} \mathcal{M}_{0}(z) u+P_{\text {ker }\left(C_{s}\right)} C_{d}^{*} q=f
$$

which yields (26). (27) follows analogously.

The next result is a reformulation of the system (26)-(27). For this, we introduce the canonical embeddings

$$
\begin{aligned}
& \iota_{s}: \operatorname{ker}\left(C_{s}\right) \hookrightarrow\left(H_{d} \otimes H_{s}\right)^{m}, \\
& \iota_{s^{*}}: \operatorname{ker}\left(C_{s}^{*}\right) \hookrightarrow\left(H_{d} \otimes H_{s}\right)^{n} .
\end{aligned}
$$

Since $u=\iota_{s} \iota_{s}^{*} u$ and $q=\iota_{s^{*}} \iota_{s^{*}}^{*} q$, we obtain the following.

Corollary 4.8. Let $(u, q)$ satisfy the system (26)-(27). Then

$$
\begin{aligned}
\iota_{s}^{*} \mathcal{M}_{0}(z) \iota_{s} \iota_{s}^{*} u+\iota_{s}^{*} C_{d}^{*} \iota_{s^{*}} \iota_{s^{*}}^{*} q & =\iota_{s}^{*} f, \\
\iota_{s^{*}}^{*} M_{1}(z) \iota_{s^{*}} \iota_{s^{*}}^{*} q-\iota_{s^{*}}^{*} C_{d} \iota_{s} \iota_{s}^{*} u & =\iota_{s^{*}}^{*} g .
\end{aligned}
$$

Remark 4.9. The equations for $u$ and $q$ from Corollary 4.8 can be written in the following block operator matrix form

$$
\left(\left(\begin{array}{cc}
\widetilde{\mathcal{M}}_{0}(z) & 0 \\
0 & \widetilde{\mathcal{M}}_{1}(z)
\end{array}\right)+\left(\begin{array}{cc}
0 & \iota_{s}^{*} C_{d}^{*} \iota_{s^{*}} \\
-\iota_{s^{*}}^{*} C_{d} \iota_{s} & 0
\end{array}\right)\right)\left(\begin{array}{l}
\tilde{u} \\
\tilde{q}
\end{array}\right)=\left(\begin{array}{l}
\tilde{f} \\
\tilde{g}
\end{array}\right),
$$

where $\widetilde{\mathcal{M}}_{0}(z)=\iota_{s}^{*} \mathcal{M}_{0}(z) \iota_{s}, \widetilde{\mathcal{M}}_{1}(z)=\iota_{s^{*}}^{*} \mathcal{M}_{1}(z) \iota_{s^{*}}, \tilde{u}=\iota_{s}^{*} u, \tilde{q}=\iota_{s^{*}}^{*} q, \tilde{f}=\iota_{s}^{*} f$ and $\tilde{g}=\iota_{s^{*}}^{*} g$.

From this remark we obtain the homogenized problem as follows. Namely,

$$
\left(\left(\begin{array}{cc}
\widetilde{\mathcal{M}}_{0}(z) & 0 \\
0 & \widetilde{\mathcal{M}}_{1}(z)
\end{array}\right)+\left(\begin{array}{cc}
0 & \widetilde{C}_{d}^{*} \\
-\widetilde{C}_{d} & 0
\end{array}\right)\right)\left(\begin{array}{l}
w \\
r
\end{array}\right)=\left(\begin{array}{l}
h \\
j
\end{array}\right)
$$

where $\widetilde{C_{d}}=\overline{\iota_{s^{*}}^{*} C_{d} \iota_{s}}$ and ${\widetilde{C_{d}}}^{*}=\overline{\iota_{s}^{*} C_{d}^{*} \iota_{s^{*}}}$. In particular, we deduce that $(\tilde{u}, \tilde{q})$ is uniquely determined, see [34, Lemma 2.12].

This observation combined with Corollary 4.8 yields that the claim of Theorem 4.7 is true even without choosing subsequences. In any case, we can formulate the following homogenization result for time-dependent homogenization problems, which is one of the main results of this article. We define $\mathcal{S}_{\varepsilon}(z) \in L(\mathcal{H}, H)$ by

$$
\mathcal{S}_{\varepsilon}(z)(f, g):=S_{\varepsilon}(z)\left(\begin{array}{l}
f \\
g
\end{array}\right)
$$

where $S_{\varepsilon}(z)$ is given by $(25)$. We shall also define $\mathcal{S}_{0}(z) \in L(\mathcal{H}, H)$ via

$$
\mathcal{S}_{0}(z)(f, g):=\left(\begin{array}{cc}
\iota_{s} & 0 \\
0 & \iota_{s^{*}}
\end{array}\right)\left(\left(\begin{array}{cc}
\widetilde{\mathcal{M}}_{0}(z) & 0 \\
0 & \widetilde{\mathcal{M}}_{1}(z)
\end{array}\right)+\left(\begin{array}{cc}
0 & \widetilde{C}_{d}^{*} \\
-\widetilde{C}_{d} & 0
\end{array}\right)\right)^{-1}\left(\begin{array}{cc}
\iota_{s}^{*} & 0 \\
0 & \iota_{s^{*}}^{*}
\end{array}\right)\left(\begin{array}{l}
f \\
g
\end{array}\right) .
$$

Theorem 4.10. Assume (10)-(13) and that $H$ is separable. Then we have

$$
\mathcal{T}_{\varepsilon} \mathcal{S}_{\varepsilon}\left(\partial_{t, \nu}\right) \rightarrow \mathcal{S}_{0}\left(\partial_{t, \nu}\right) \quad(\varepsilon \rightarrow 0)
$$

in the weak operator topology of $L\left(L_{\nu}^{2}(\mathbb{R} ; \mathcal{H}), L_{\nu}^{2}(\mathbb{R} ; H)\right)$.

Proof. For $z \in \mathbb{C}_{\operatorname{Re}>\nu_{0}}$ we obtain with Theorem 4.7 (in the version without choosing subsequences, which is justified by the subsequence principle in combination with Corollary 4.8 and the representation in Remark 4.9) that $\mathcal{T}_{\varepsilon} \mathcal{S}_{\varepsilon}(z) \rightarrow \mathcal{S}_{0}(z)$ in the weak operator topology of $L(\mathcal{H}, H)$. Thus, the assertion follows from Theorem 4.3. 


\section{$5 \quad$ Examples}

In this section we present two specific examples of the unfolding operator - the stochastic and (a variant of the) periodic unfolding operator. Moreover, we provide specific examples in which Theorem 3.1 and Theorem 4.10 yield homogenization results. In particular, we consider homogenization problems for elliptic, Maxwell's and wave equations. Also, besides the essential homogenization results obtained by Theorem 4.10 for the evolutionary equations, with little additional effort we prove some corrector type results.

\subsection{Examples of unfolding operators}

Deterministic differential operators. First, we introduce the deterministic differential operators which we use in the description of the considered problems. In the following, we denote by $\partial_{i}$, $\nabla=\left(\partial_{1}, \ldots, \partial_{n}\right)$, and div the partial derivative, the gradient and the divergence, respectively, and use the notation

$$
\nabla \times u:=\left(\partial_{2} u_{3}-\partial_{3} u_{2}, \partial_{3} u_{1}-\partial_{1} u_{3}, \partial_{1} u_{2}-\partial_{2} u_{1}\right)
$$

for the curl of a smooth three-dimensional vector-field. Let $Q \subseteq \mathbb{R}^{n}$ be open.

Gradient and divergence operators. The operator closure of $C_{c}^{\infty}(Q) \subseteq L^{2}(Q) \rightarrow L^{2}(Q)^{n}, \varphi \mapsto \nabla \varphi$ and its adjoint are denoted by

$$
\stackrel{\circ}{\operatorname{grad}_{x}}: \operatorname{dom}\left(\operatorname{grad}_{x}\right)=H_{0}^{1}(Q) \subseteq L^{2}(Q) \rightarrow L^{2}(Q)^{n} \quad \text { and } \quad-\operatorname{div}_{x}=\left(\stackrel{\circ}{\operatorname{grad}_{x}}\right)^{*} .
$$

Curl operator. Let $n=3$. The operator closure of $C_{c}^{\infty}(Q)^{3} \subseteq L^{2}(Q)^{3} \rightarrow L^{2}(Q)^{3}, u \mapsto \nabla \times u$ and its adjoint are denoted by

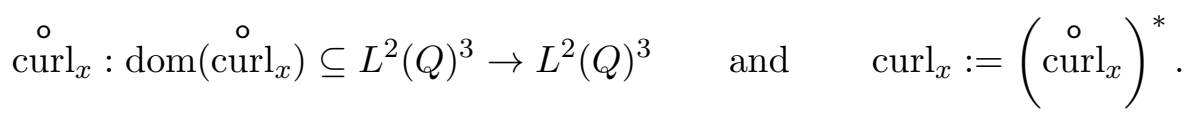

\section{Periodic unfolding (in the mean)}

We present a periodic unfolding operator suited for homogenization problems involving periodic coefficients. We consider the unit torus $\square:=\mathbb{R}^{n} / \mathbb{Z}^{n}$ equipped with the push-forward of the Lebesgue measure $\mathcal{L}\left([0,1)^{n}\right)$ and let $Q \subseteq \mathbb{R}^{n}$ be open. We consider the following particular choice of the abstract Hilbert spaces from Section 2: $H_{d}=L^{2}(Q), H_{s}=L^{2}(\square)$. For $\varepsilon \in \mathbb{R} \backslash\{0\}$, we let $\mathcal{T}_{\varepsilon}: L^{2}(Q) \stackrel{a}{\otimes} L^{2}(\square) \rightarrow L^{2}(Q) \otimes L^{2}(\square)$ be given by

$$
\mathcal{T}_{\varepsilon} u(x, y)=u\left(x, y-\left\{\frac{x}{\varepsilon}\right\}_{\square}\right),
$$

where $\{\cdot\}_{\square}: \mathbb{R}^{n} \rightarrow \square$ is the canonical quotient map. $\mathcal{T}_{\varepsilon}$ is a linear isometry and we call its continuous extension $\mathcal{T}_{\varepsilon}: L^{2}(Q) \otimes L^{2}(\square) \rightarrow L^{2}(Q) \otimes L^{2}(\square)$ periodic unfolding operator. Thus, $\mathcal{T}_{\varepsilon}$ is unitary and we have $\mathcal{T}_{\varepsilon}^{-1}=\mathcal{T}_{-\varepsilon}$.

Remark 5.1. The above notion of periodic unfolding differs from the classical notion of unfolding introduced in $[8,9]$. In particular, in $[8,9]$ the unfolding operator is defined as

$$
\widetilde{\mathcal{T}}_{\varepsilon}: L^{2}(Q) \rightarrow L^{2}(Q) \otimes L^{2}(\square), \text { given by } \widetilde{\mathcal{T}}_{\varepsilon} u(x, y)=u\left(\left[\frac{x}{\varepsilon}\right]_{\varepsilon \mathbb{Z}^{n}}+\varepsilon y\right),
$$


where $[\cdot]_{\varepsilon \mathbb{Z}^{n}}: \mathbb{R}^{n} \rightarrow \varepsilon \mathbb{Z}^{n}$ is defined as $[x]_{\varepsilon \mathbb{Z}^{n}}=x-\varepsilon\left\{\frac{x}{\varepsilon}\right\}_{\square}$. The operator $\widetilde{\mathcal{T}}_{\varepsilon}$ defined in this fashion is not surjective and therefore it does not satisfy the assumptions of our abstract setting. On the other hand, definition (30) involves an additional variable $y$ for functions in the domain of $\mathcal{T}_{\varepsilon}$ (in this respect we might call the notion defined in (30) periodic unfolding in the mean) and consequently it fulfills the requirements from Section 2.

The operator closure of $C^{\infty}(\square) \subseteq L^{2}(\square) \rightarrow L^{2}(\square)^{n}, \varphi \mapsto \nabla \varphi$ and its adjoint are denoted by

$$
\operatorname{grad}_{y}^{\#}: \operatorname{dom}\left(\operatorname{grad}_{y}^{\#}\right)=H_{\#}^{1}(\square) \subseteq L^{2}(\square) \rightarrow L^{2}(\square)^{n} \quad \text { and } \quad-\operatorname{div}_{y}^{\#}=\left(\operatorname{grad}_{y}^{\#}\right)^{*} .
$$

Note that we may identify $C^{\infty}(\square)$ with the space of $\mathbb{Z}^{n}$-periodic functions in $C^{\infty}\left(\mathbb{R}^{n}\right)$, and $L^{2}(\square)$ with $L^{2}\left((0,1)^{n}\right)$.

If we set $C_{d}=\stackrel{\circ}{\operatorname{grad}_{x}}$ and $C_{s}=\operatorname{grad}_{y}^{\#}$, it follows that the abstract conditions (12)-(14) are satisfied (Note that (10) is easy, by choosing $D_{1}=D_{2}=C_{c}^{\infty}(Q)$ and $E_{1}=E_{2}=C^{\infty}(\square)$ ):

Lemma 5.2. For $\varepsilon \neq 0$, we have

$$
\begin{array}{r}
\stackrel{\circ}{\varepsilon \operatorname{grad}_{x} \mathcal{T}_{-\varepsilon} \varphi=\varepsilon \mathcal{T}_{-\varepsilon} \operatorname{grad}_{x} \varphi+\operatorname{grad}_{y}^{\#} \mathcal{T}_{-\varepsilon} \varphi,}, \quad \text { for all } \varphi \in \operatorname{dom}\left(\operatorname{grad}_{x}\right) \cap \operatorname{dom}\left(\operatorname{grad}_{y}^{\#}\right), \\
\mathcal{T}_{\varepsilon} \operatorname{grad}_{y}^{\#} \subseteq \operatorname{grad}_{y}^{\#} \mathcal{T}_{\varepsilon}, \\
\mathcal{T}_{\varepsilon} \varphi=\varphi, \quad \text { for all } \varphi \in \operatorname{ker}\left(\operatorname{grad}_{y}^{\#}\right) .
\end{array}
$$

Note that $\operatorname{dom}\left(\stackrel{\circ}{\operatorname{grad}_{x}}\right) \cap \operatorname{dom}\left(\operatorname{grad}_{y}^{\#}\right)=H_{0}^{1}(Q) \otimes L^{2}(\square) \cap L^{2}(Q) \otimes H_{\#}^{1}(\square)$ and $\operatorname{ker}\left(\operatorname{grad}_{y}^{\#}\right) \simeq$ $L^{2}(Q) \otimes \mathbb{C}$.

Proof of Lemma 5.2. Let $\eta \in C_{c}^{\infty}(Q) \stackrel{a}{\otimes} C^{\infty}(\square)^{n}$. Then

$$
\left\langle\varepsilon \mathcal{T}_{-\varepsilon}^{\left.\operatorname{grad}_{x} \varphi, \eta\right\rangle_{L^{2}(Q) \otimes L^{2}(\square)^{n}}^{\circ}}=-\left\langle\varphi, \varepsilon \operatorname{div}_{x} \mathcal{T}_{\varepsilon} \eta\right\rangle_{L^{2}(Q) \otimes L^{2}(\square)} .\right.
$$

Using the smoothness of $\eta$ and the chain rule, we compute $\varepsilon \operatorname{div}_{x} \mathcal{T}_{\varepsilon} \eta=\varepsilon \mathcal{T}_{\varepsilon} \operatorname{div}_{x} \eta-\mathcal{T}_{\varepsilon} \operatorname{div}_{y}^{\#} \eta$, where we use that $\operatorname{div}_{x} \mathcal{T}_{\varepsilon} \eta(x, y)=\partial_{x_{i}}\left(\eta_{i}\left(x, y-\left\{\frac{x}{\varepsilon}\right\}_{\square}\right)\right)$ a.e. Therefore, we obtain

$$
\left\langle\varepsilon \mathcal{T}_{-\varepsilon} \operatorname{grad}_{x} \varphi, \eta\right\rangle_{L^{2}(Q) \otimes L^{2}(\square)^{n}}=\left\langle\operatorname{\varepsilon grad}_{x} \mathcal{T}_{-\varepsilon} \varphi-\operatorname{grad}_{y}^{\#} \mathcal{T}_{-\varepsilon} \varphi, \eta\right\rangle_{L^{2}(Q) \otimes L^{2}(\square)^{n}} .
$$

Since $C_{c}^{\infty}(Q) \stackrel{a}{\otimes} C^{\infty}(\square)$ is dense in $\operatorname{dom}\left(\stackrel{\circ}{\operatorname{grad}_{x}}\right) \cap \operatorname{dom}\left(\operatorname{grad}_{y}^{\#}\right)$, the first claim follows.

The second claim follows from the fact that $\mathcal{T}_{\varepsilon} \operatorname{grad}_{y}^{\#} \varphi=\operatorname{grad}_{y}^{\#} \mathcal{T}_{\varepsilon} \varphi$ for any $\varphi \in C^{\infty}(\square)$.

The last claim follows using that $\operatorname{ker}\left(\operatorname{grad}_{y}^{\#}\right) \simeq L^{2}(Q) \otimes \mathbb{C}$, which can be obtained with the help of the Poincaré inequality: There exists $C>0$ such that

$$
\left\|\varphi-\int_{\square} \varphi\right\|_{L^{2}(\square)} \leqslant C\left\|\operatorname{grad}_{y}^{\#} \varphi\right\|_{L^{2}(\square)^{n}} \quad \text { for all } \varphi \in \operatorname{dom}\left(\operatorname{grad}_{y}^{\#}\right) .
$$




\section{Stochastic unfolding}

Let $Q \subseteq \mathbb{R}^{n}$ be open, $(\Omega, \Sigma, \mu, \tau)$ be a probability space satisfying Assumption 1 . In this section, we set $H_{d}=L^{2}(Q)$ and $H_{s}=L^{2}(\Omega)$ and consider the stochastic unfolding operator $\mathcal{T}_{\varepsilon}: L^{2}(Q) \otimes L^{2}(\Omega) \rightarrow$ $L^{2}(Q) \otimes L^{2}(\Omega)$ defined in $(7)$.

Remark 5.3. The stochastic unfolding operator is a generalization of the periodic unfolding operator since in the case $(\Omega, \Sigma, \mu)=(\square, \mathcal{L}, d y)$ and $\tau_{x} y=y+\{x\}_{\square}$ the two operators coincide. Another instance of stochastic unfolding corresponds to the choice $(\Omega, \Sigma, \mu)=\left(\square^{m}, \mathcal{L}^{m},(d y)^{m}\right.$ ) (for some $m \in \mathbb{N})$ with $\tau_{x} y=\left(y_{1}+\{x\}_{\square}, \ldots, y_{m}+\{x\}_{\square}\right)$. The latter is well-suited for the treatment of problems involving quasi-periodic rapidly-oscillating coefficients.

With help of the dynamical system $\tau$ (for fixed $i \in\{1, \ldots, n\}$ ) we introduce the group of unitary operators $(h \in \mathbb{R}) T_{h e_{i}}: L^{2}(\Omega) \rightarrow L^{2}(\Omega)$ given by

$$
T_{h e_{i}} \varphi=\varphi \circ \tau_{h e_{i}} .
$$

This group is strongly continuous and we denote its infinitesimal generator by $\partial_{\omega, i}: \operatorname{dom}\left(\partial_{\omega, i}\right) \subseteq$ $L^{2}(\Omega) \rightarrow L^{2}(\Omega)$, i.e.,

$$
\partial_{\omega, i} \varphi=\lim _{h \rightarrow 0} \frac{T_{h e_{i}} \varphi-\varphi}{h} .
$$

Analogously to (31), we define $T_{x}: L^{2}(\Omega) \rightarrow L^{2}(\Omega)$ for $x \in \mathbb{R}^{n}$. Using the stochastic partial derivatives $\partial_{\omega, i}$, we define the stochastic gradient

$$
\operatorname{grad}_{\omega}: H^{1}(\Omega):=\bigcap_{i \in\{1, \ldots, n\}} \operatorname{dom}\left(\partial_{\omega, i}\right) \subseteq L^{2}(\Omega) \rightarrow L^{2}(\Omega)^{n}
$$

given by

$$
\operatorname{grad}_{\omega} \varphi=\left(\partial_{\omega, 1} \varphi, \ldots, \partial_{\omega, n} \varphi\right) .
$$

It is a closed and densely defined linear operator and we let $\operatorname{div}_{\omega}=-\left(\operatorname{grad}_{\omega}\right)^{*}$. Also, we introduce the space of smooth random variables by

$$
H^{\infty}(\Omega)=\left\{\varphi \in L^{2}(\Omega): \partial_{\omega, 1}^{\alpha_{1}} \cdots \partial_{\omega, n}^{\alpha_{n}} \varphi \in H^{1}(\Omega) \text { for all } \alpha_{1}, \ldots, \alpha_{n} \in \mathbb{N}_{0}\right\} .
$$

In the case $n=3$, we define the stochastic counterpart of the $\stackrel{\circ}{\operatorname{corl}} x$ operator as follows. Let $\nabla_{\omega} \times: H^{\infty}(\Omega)^{3} \subseteq L^{2}(\Omega)^{3} \rightarrow L^{2}(\Omega)^{3}$ be given by

$$
\nabla_{\omega} \times \varphi=\left(\partial_{\omega, 2} \varphi_{3}-\partial_{\omega, 3} \varphi_{2}, \partial_{\omega, 3} \varphi_{1}-\partial_{\omega, 1} \varphi_{3}, \partial_{\omega, 1} \varphi_{2}-\partial_{\omega, 2} \varphi_{1}\right)
$$

We let $\operatorname{curl}_{\omega}=\left(\nabla_{\omega} \times\right)^{* *}$. The choice $C_{d}=\stackrel{\circ}{\operatorname{grad}_{x}}$ and $C_{s}=\operatorname{grad}_{\omega}$ satisfies assumptions (12)-(14), and if we set $C_{d}=\operatorname{curl}_{x}$ and $C_{s}=\operatorname{curl}_{\omega}$ we merely obtain (12)-(13). (Again, note that (10) in both cases follows with the choice $D_{1}=D_{2}=C_{c}^{\infty}(Q)$ and $E_{1}=E_{2}=H^{\infty}(\Omega)$.)

Lemma 5.4. Let $\varepsilon \neq 0$.

(a) Then

$$
\begin{gathered}
\stackrel{\circ}{\operatorname{grad}_{x} \mathcal{T}_{-\varepsilon} \varphi=\varepsilon \mathcal{T}_{-\varepsilon} \operatorname{grad}_{x} \varphi+\operatorname{grad}_{\omega} \mathcal{T}_{-\varepsilon} \varphi} \begin{array}{c}
\circ \\
\mathcal{T}_{\varepsilon} \operatorname{grad}_{\omega} \subseteq \\
\operatorname{grad}_{\omega} \mathcal{T}_{\varepsilon},
\end{array} \\
\mathcal{T}_{\varepsilon} \varphi=\varphi
\end{gathered}
$$


(b) If $n=3$, then

$$
\begin{gathered}
\stackrel{\circ}{\operatorname{curl}_{x} \mathcal{T}_{-\varepsilon} \varphi=\varepsilon \mathcal{T}_{-\varepsilon}} \stackrel{\circ}{\operatorname{curl}_{x} \varphi+\operatorname{curl}_{\omega} \mathcal{T}_{-\varepsilon} \varphi} \quad \text { for all } \varphi \in \operatorname{dom}\left(\operatorname{corl}_{x}\right) \cap \operatorname{dom}\left(\operatorname{curl}_{\omega}\right), \\
\mathcal{T}_{\varepsilon} \operatorname{curl}_{\omega} \subseteq \operatorname{curl}_{\omega} \mathcal{T}_{\varepsilon} .
\end{gathered}
$$

Proof. (a) Let $\eta \in C_{c}^{\infty}(Q) \stackrel{a}{\otimes} H^{\infty}(\Omega)^{n}$. We have

$$
\left\langle\varepsilon \mathcal{T}_{-\varepsilon} \operatorname{orad}_{x} \varphi, \eta\right\rangle_{L^{2}(Q) \otimes L^{2}(\Omega)}=\left\langle\varphi, \varepsilon \operatorname{div}_{x} \mathcal{T}_{\varepsilon} \eta\right\rangle_{L^{2}(Q) \otimes L^{2}(\Omega)} .
$$

We compute $\varepsilon \operatorname{div}_{x} \mathcal{T}_{\varepsilon} \eta=\varepsilon \mathcal{T}_{\varepsilon} \operatorname{div}_{x} \eta-\mathcal{T}_{\varepsilon} \operatorname{div}_{\omega} \eta$, in order to obtain,

$$
\left\langle\varepsilon \mathcal{T}_{-\varepsilon}^{\left.\stackrel{\circ}{\operatorname{grad}_{x} \varphi, \eta}\right\rangle_{L^{2}(Q) \otimes L^{2}(\Omega)}}=\left\langle\operatorname{\varepsilon grad}_{x}^{\circ} \mathcal{T}_{-\varepsilon} \varphi-\operatorname{grad}_{\omega} \mathcal{T}_{-\varepsilon} \varphi, \eta\right\rangle_{L^{2}(Q) \otimes L^{2}(\Omega)} .\right.
$$

Note that $C_{c}^{\infty}(Q) \stackrel{a}{\otimes} H^{\infty}(\Omega)$ is dense in $\operatorname{dom}\left(\operatorname{grad}_{x}\right) \cap \operatorname{dom}\left(\operatorname{grad}_{\omega}\right)$ (see (33) below) and therefore the first claim follows.

In order to obtain the second claim, it is sufficient to show that $\mathcal{T}_{\varepsilon} \partial_{\omega, i} \varphi=\partial_{\omega, i} \mathcal{T}_{\varepsilon} \varphi$ for any $\varphi \in H^{\infty}(\Omega)$ (for any $i \in\{1, \ldots, n\}$ ). Let $\varphi, \eta \in L^{2}(Q) \stackrel{a}{\otimes} H^{\infty}(\Omega)$. We have

$$
\begin{aligned}
\left\langle\mathcal{T}_{\varepsilon} \partial_{\omega, i} \varphi, \eta\right\rangle_{L^{2}(Q) \otimes L^{2}(\Omega)} & =\lim _{h \rightarrow 0} \frac{1}{h}\left\langle\mathcal{T}_{\varepsilon} T_{h e_{i}} \varphi-\mathcal{T}_{\varepsilon} \varphi, \eta\right\rangle_{L^{2}(Q) \otimes L^{2}(\Omega)} \\
& =\lim _{h \rightarrow 0} \frac{1}{h}\left\langle\mathcal{T}_{\varepsilon} \varphi, T_{-h e_{i}} \eta-\eta\right\rangle_{L^{2}(Q) \otimes L^{2}(\Omega)} \\
& =-\left\langle\mathcal{T}_{\varepsilon} \varphi, \partial_{\omega, i} \eta\right\rangle_{L^{2}(Q) \otimes L^{2}(\Omega)} \\
& =\left\langle\partial_{\omega, i} \mathcal{T}_{\varepsilon} \varphi, \eta\right\rangle_{L^{2}(Q) \otimes L^{2}(\Omega)}
\end{aligned}
$$

As a result of this and by the density of $H^{\infty}(\Omega)$ in $H^{1}(\Omega)$, the claim follows.

For $\varphi \in \operatorname{ker}\left(\operatorname{grad}_{\omega}\right)$, we have $T_{x} \varphi=\varphi$ for all $x \in \mathbb{R}^{n}$ (see, e.g., [14, Lemma 3.10]). As a result of this, the third claim follows.

(b) The proof follows analogously to part (a).

Remark 5.5 (Boundary conditions). The first commutation relation from Lemmas 5.2 and 5.4 (a) remains valid if the gradient operator $\operatorname{grad}_{x}$ (which is defined on a domain which accounts for homogeneous Dirichlet boundary conditions) is replaced by gradient operators corresponding to other types of boundary conditions (e.g., with homogeneous Neumann condition or periodic boundary condition). In this respect, the unfolding procedure does not only apply to problems with certain boundary conditions.

Auxiliary results. We provide certain facts that will be helpful in the treatment of the stochastic homogenization problems considered in the following section (in particular for corrector type results). The following standard orthogonal decompositions hold (see, e.g., [7])

$$
L^{2}(\Omega)=\operatorname{ker}\left(\operatorname{grad}_{\omega}\right) \oplus^{\perp} \overline{\operatorname{ran}}\left(\operatorname{div}_{\omega}\right), \quad L^{2}(\Omega)^{n}=\operatorname{ker}\left(\operatorname{div}_{\omega}\right) \oplus^{\perp} \overline{\operatorname{ran}}\left(\operatorname{grad}_{\omega}\right) .
$$

$P_{\text {inv }}$ and $P_{\text {pot }}$ denote the orthogonal projections $P_{\text {inv }}: L^{2}(\Omega) \rightarrow \operatorname{ker}\left(\operatorname{grad}_{\omega}\right)$ and $P_{\text {pot }}: L^{2}(\Omega)^{n} \rightarrow$ $\overline{\operatorname{ran}}\left(\operatorname{grad}_{\omega}\right)=: L_{\text {pot }}^{2}(\Omega)$. We have $\operatorname{ker}\left(\operatorname{grad}_{\omega}\right)=\left\{u \in L^{2}(\Omega): T_{x} u=u\right.$ for all $\left.x \in \mathbb{R}^{n}\right\}=: L_{\text {inv }}^{2}(\Omega)$ (see, e.g., [14, Lemma 3.10]). If we additionally assume that the probability space is ergodic, we get $L_{\text {inv }}^{2}(\Omega) \simeq \mathbb{C}$. 
Lemma 5.6. Let $n=3$. Then

$$
\operatorname{ker}\left(\operatorname{curl}_{\omega}\right)=L_{\text {inv }}^{2}(\Omega)^{3} \oplus^{\perp} L_{\text {pot }}^{2}(\Omega), \quad \operatorname{ker}\left(\operatorname{curl}_{\omega}^{*}\right)=L_{\text {inv }}^{2}(\Omega)^{3} \oplus^{\perp} L_{\text {pot }}^{2}(\Omega) .
$$

The following standard mollification procedure for random variables (see, e.g., $[15,4]$ ) is useful in the proof of the above lemma. For a sequence $\delta \rightarrow 0$, we consider a sequence of standard mollifiers $\rho_{\delta} \in C_{c}^{\infty}\left(\mathbb{R}^{n}\right)$ (even and non-negative) and for $\varphi \in L^{2}(\Omega)$, we set

$$
\varphi_{\delta}=\int_{\mathbb{R}^{n}} \rho_{\delta}(y) T_{y} \varphi d y .
$$

We obtain that $\varphi_{\delta} \in H^{\infty}(\Omega)$ (apply the definition of $\partial_{\omega, i}$, transform the integral to have the difference quotient on $\rho_{\delta}$, and then apply the dominated convergence theorem), $\left\langle\varphi_{\delta}\right\rangle=\langle\varphi\rangle=$ $\int_{\Omega} \varphi(\omega) d \mu(\omega)$ and $\varphi_{\delta} \rightarrow \varphi$ in $L^{2}(\Omega)$ as $\delta \rightarrow 0$ (see, e.g., [15, Section 7.2]). We collect some further useful properties of this mollification procedure:

Lemma 5.7. Let $\varphi \in L^{2}(\Omega)$. Then $\varphi_{\delta} \in H^{\infty}(\Omega)$; and $\varphi_{\delta} \rightarrow \varphi$ in $L^{2}(\Omega)$ as $\delta \rightarrow 0$. Moreover, the following statements hold:

(a) If $\varphi \in H^{1}(\Omega)$, then $\operatorname{grad}_{\omega} \varphi_{\delta}=\left(\operatorname{grad}_{\omega} \varphi\right)_{\delta}$ and $\operatorname{grad}_{\omega} \varphi_{\delta} \rightarrow \operatorname{grad}_{\omega} \varphi$.

(b) If $\varphi \in \operatorname{dom}\left(\operatorname{div}_{\omega}\right)$, then $\operatorname{div}_{\omega} \varphi_{\delta}=\left(\operatorname{div}_{\omega} \varphi\right)_{\delta}$ and $\operatorname{div}_{\omega} \varphi_{\delta} \rightarrow \operatorname{div}_{\omega} \varphi$.

(c) $(n=3)$ If $\varphi \in \operatorname{dom}\left(\operatorname{curl}_{\omega}\right)$, then $\operatorname{curl}_{\omega} \varphi_{\delta}=\left(\operatorname{curl}_{\omega} \varphi\right)_{\delta}$ and $\operatorname{curl}_{\omega} \varphi_{\delta} \rightarrow \operatorname{curl}_{\omega} \varphi$.

(d) $(n=3)$ If $\varphi \in \operatorname{dom}\left(\operatorname{curl}_{\omega}^{*}\right)$, then $\operatorname{curl}_{\omega}^{*} \varphi_{\delta}=\left(\operatorname{curl}_{\omega}^{*} \varphi\right)_{\delta}$ and $\operatorname{curl}_{\omega}^{*} \varphi_{\delta} \rightarrow \operatorname{curl}_{\omega}^{*} \varphi$.

(e) $\operatorname{curl}_{\omega}=\operatorname{curl}_{\omega}^{*}$.

Proof. The first statements of the lemma follow the same way as they follow in the deterministic case.

(a) We have (for $i \in\{1, \ldots, n\}$ ),

$$
\left\|\frac{1}{h}\left(T_{h e_{i}} \varphi_{\delta}-\varphi_{\delta}\right)-\left(\partial_{\omega, i} \varphi\right)_{\delta}\right\|_{L^{2}(\Omega)}=\left\|\int_{\mathbb{R}^{n}} \rho_{\delta}(y)\left(\frac{T_{y+h e_{i}} \varphi-T_{y} \varphi}{h}-T_{y} \partial_{\omega, i} \varphi\right)\right\|_{L^{2}(\Omega)} .
$$

Using the above and that $\rho_{\delta}$ is bounded and compactly supported, we obtain that there exists $C(\delta) \geqslant 0$ such that

$$
\left\|\frac{1}{h}\left(T_{h e_{i}} \varphi_{\delta}-\varphi_{\delta}\right)-\left(\partial_{\omega, i} \varphi\right)_{\delta}\right\|_{L^{2}(\Omega)} \leqslant C(\delta)\left\|\frac{1}{h}\left(T_{h e_{i}} \varphi-\varphi\right)-\partial_{\omega, i} \varphi\right\|_{L^{2}(\Omega)} .
$$

In the limit $h \rightarrow 0$ the right-hand side vanishes, and this implies that $\operatorname{grad}_{\omega} \varphi_{\delta}=\left(\operatorname{grad}_{\omega} \varphi\right)_{\delta}$. Consequently, $\operatorname{grad}_{\omega} \varphi_{\delta} \rightarrow \operatorname{grad}_{\omega} \varphi$.

(b) For $\eta \in H^{1}(\Omega)$, we have

$$
-\left\langle\varphi_{\delta}, \operatorname{grad}_{\omega} \eta\right\rangle_{L^{2}(\Omega)^{n}}=-\left\langle\int_{\mathbb{R}^{n}} \rho_{\delta}(y) T_{y} \varphi \operatorname{grad}_{\omega} \eta d y\right\rangle=-\int_{\mathbb{R}^{n}}\left\langle\rho_{\delta}(y) T_{y} \varphi \operatorname{grad}_{\omega} \eta\right\rangle d y .
$$

Using that $T_{-y}$ and (the infinitesimal generator) $\operatorname{grad}_{\omega}$ commute, we obtain that the last expression equals

$$
-\int_{\mathbb{R}^{n}} \rho_{\delta}(y)\left\langle\varphi \operatorname{grad}_{\omega} T_{-y} \eta\right\rangle d y=\left\langle\int_{\mathbb{R}^{n}} \rho_{\delta}(y) T_{y} \operatorname{div}_{\omega} \varphi d y \eta\right\rangle=\left\langle\left(\operatorname{div}_{\omega} \varphi\right)_{\delta}, \eta\right\rangle_{L^{2}(\Omega)} .
$$


As a result of this, we have $\operatorname{div}_{\omega} \varphi_{\delta}=\left(\operatorname{div}_{\omega} \varphi\right)_{\delta}$ and $\operatorname{div}_{\omega} \varphi_{\delta} \rightarrow \operatorname{div}_{\omega} \varphi$.

(c) Let $\eta \in \operatorname{dom}\left(\operatorname{curl}_{\omega}^{*}\right)$. We have

$$
\left\langle\varphi_{\delta}, \operatorname{curl}_{\omega}^{*} \eta\right\rangle_{L^{2}(\Omega)^{3}}=\left\langle\int_{\mathbb{R}^{n}} \rho_{\delta}(y) T_{y} \varphi \operatorname{curl}_{\omega}^{*} \eta d y\right\rangle=\int_{\mathbb{R}^{n}} \rho_{\delta}(y)\left\langle\varphi T_{-y} \operatorname{curl}_{\omega}^{*} \eta\right\rangle d y .
$$

In order to obtain the claim of the lemma, it is sufficient to show that $T_{-y} \operatorname{curl}_{\omega}^{*} \eta=\operatorname{curl}_{\omega}^{*} T_{-y} \eta$. Indeed, in that case the above expression equals

$$
\int_{\mathbb{R}^{n}} \rho_{\delta}(y)\left\langle\varphi \operatorname{curl}_{\omega}^{*} T_{-y} \eta\right\rangle d y=\left\langle\int_{\mathbb{R}^{n}} \rho_{\delta}(y) T_{y} \operatorname{curl}_{\omega} \varphi d y \eta\right\rangle=\left\langle\left(\operatorname{curl}_{\omega} \varphi\right)_{\delta}, \eta\right\rangle_{L^{2}(\Omega)^{3}},
$$

and therefore the claim follows. In the following we show that $T_{-y} \operatorname{curl}_{\omega}^{*} \eta=\operatorname{curl}_{\omega}^{*} T_{-y} \eta$. Let $\psi \in H^{1}(\Omega)^{3}$. We have

$$
\left\langle T_{-y} \eta, \operatorname{curl}_{\omega} \psi\right\rangle_{L^{2}(\Omega)^{3}}=\left\langle\eta, T_{y} \operatorname{curl}_{\omega} \psi\right\rangle_{L^{2}(\Omega)^{3}}=\left\langle\eta, \operatorname{curl}_{\omega} T_{y} \psi\right\rangle_{L^{2}(\Omega)^{3}}=\left\langle T_{-y} \operatorname{curl}_{\omega}^{*} \eta, \psi\right\rangle_{L^{2}(\Omega)^{3}}
$$

By density of $H^{1}(\Omega)^{3}$ in $\operatorname{dom}\left(\operatorname{curl}_{\omega}\right)$, we obtain that for any $\psi \in \operatorname{dom}\left(\operatorname{curl}_{\omega}\right),\left\langle T_{-y} \eta, \operatorname{curl}_{\omega} \psi\right\rangle_{L^{2}(\Omega)^{3}}=$ $\left\langle T_{-y} \operatorname{curl}_{\omega}^{*} \eta, \psi\right\rangle_{L^{2}(\Omega)^{3}}$ that implies $T_{-y} \eta \in \operatorname{dom}\left(\operatorname{curl}_{\omega}^{*}\right)$ and $T_{-y} \operatorname{curl}_{\omega}^{*} \eta=\operatorname{curl}_{\omega}^{*} T_{-y} \eta$. The proof is done.

(d) The proof follows analogously to part (c) if we obtain that for $\eta \in \operatorname{dom}\left(\operatorname{curl}_{\omega}\right), T_{-y} \operatorname{curl}_{\omega} \eta=$ $\operatorname{curl}_{\omega} T_{-y} \eta$. In order to show this, we consider a sequence $\left(\eta_{k}\right)_{k}$ in $H^{1}(\Omega)^{3}$ such that $\eta_{k} \rightarrow \eta$ and $\operatorname{curl}_{\omega} \eta_{k} \rightarrow \operatorname{curl}_{\omega} \eta$ (by definition such a sequence exists). The operator $T_{-y}$ is unitary and therefore we have $T_{-y} \eta_{k} \rightarrow T_{-y} \eta$ and $T_{-y} \operatorname{curl}_{\omega} \eta_{k} \rightarrow T_{-y} \operatorname{curl}_{\omega} \eta$. Moreover, since $T_{-y} \operatorname{curl}_{\omega} \eta_{k}=\operatorname{curl}_{\omega} T_{-y} \eta_{k}$, and using that $\operatorname{curl}_{\omega}$ is closed, we get $\operatorname{curl}_{\omega} T_{-y} \eta=T_{-y} \operatorname{curl}_{\omega} \eta$.

(e) It is elementary to show that $\operatorname{curl}_{\omega} \subseteq \operatorname{curl}_{\omega}^{*}$; see also [4, p 22]. The remaining inclusion follows from $(d)$ as this statement shows that $H^{1}(\Omega)$ is a core for $\operatorname{curl}_{\omega}^{*}$. Hence,

$$
\operatorname{curl}_{\omega} \subseteq \operatorname{curl}_{\omega}^{*}=\overline{\left.\operatorname{curl}_{\omega}^{*}\right|_{H^{1}(\Omega)}}=\overline{\left.\operatorname{curl}_{\omega}\right|_{H^{1}(\Omega)}}=\operatorname{curl}_{\omega} .
$$

Proof of Lemma 5.6. Let $\varphi \in \operatorname{ker}\left(\operatorname{curl}_{\omega}\right)$. Then $\varphi=P_{\operatorname{inv}} \varphi+\Psi$ where $\Psi=\varphi-P_{\text {inv }} \varphi$. Using (32) it follows that $\Psi$ may be decomposed as follows

$$
\Psi=\Psi_{1}+\Psi_{2},
$$

where $\Psi_{1} \in \operatorname{ker}\left(\operatorname{div}_{\omega}\right)$ and $\Psi_{2} \in \overline{\operatorname{ran}}\left(\operatorname{grad}_{\omega}\right)$. In the following we show that $\Psi_{1}=0$. Using Lemma 5.7 we find a sequence $\left(\varphi_{k}\right)_{k}$ in $H^{\infty}(\Omega)$ such that $\operatorname{grad}_{\omega} \varphi_{k} \rightarrow \Psi_{2}$ as $k \rightarrow \infty$. Moreover, since $\operatorname{curl}_{\omega} \operatorname{grad}_{\omega} \varphi_{k}=0$ for all $k \in \mathbb{N}$, we conclude that $\Psi_{2} \in \operatorname{ker}\left(\operatorname{curl}_{\omega}\right)$ and therefore it follows that

$$
\Psi_{1}=\Psi-\Psi_{2}=\varphi-P_{\mathrm{inv}} \varphi-\Psi_{2} \in \operatorname{ker}\left(\operatorname{curl}_{\omega}\right) .
$$

For the mollified functions $\Psi_{1, \delta}$ (defined as in (33)), by a direct computation we obtain

$$
\int_{\Omega}\left|\operatorname{curl}_{\omega} \Psi_{1, \delta}\right|^{2}+\left|\operatorname{div}_{\omega} \Psi_{1, \delta}\right|^{2}=\int_{\Omega}\left|\operatorname{grad}_{\omega} \Psi_{1, \delta}\right|^{2} .
$$

Using Lemma 5.7 we pass to the limit $\delta \rightarrow 0$ and it follows that $\operatorname{grad}_{\omega} \Psi_{1, \delta} \rightarrow 0$. Moreover, since $\Psi_{1, \delta} \rightarrow \Psi_{1}$, we obtain that $\operatorname{grad}_{\omega} \Psi_{1}=0$ and therefore $\Psi_{1}=0$ (using that $P_{\text {inv }} \Psi_{1}=0$ ). This concludes the proof of the first part. The second claim follows from Lemma $5.7(e)$. 


\subsection{Periodic homogenization of elliptic equations}

We start by showing that the classical example of periodic homogenization of elliptic equations fits into the previously described abstract framework (see Section 3). We refer to [3, 1] for the standard treatment of elliptic equations with periodic coefficients and to $[25,4]$ for its stochastic counterpart. Let $Q \subset \mathbb{R}^{n}$ be open and bounded. In this section, we consider the periodic unfolding operator $\mathcal{T}_{\varepsilon}: L^{2}(Q) \otimes L^{2}(\square) \rightarrow L^{2}(Q) \otimes L^{2}(\square)$ defined in (30).

In this setting the role of $C_{d}$ and $C_{s}$ is played by $\stackrel{\circ}{\operatorname{grad}_{x}}$ and $\operatorname{grad}_{y}^{\#}$, respectively. Let $A \in$ $L^{\infty}(Q \times \square)^{n \times n}$ be such that there exists $c>0$ with $|A(x, y)| \leqslant \frac{1}{c}$ and $\frac{1}{2}\left(A(x, y)+A(x, y)^{*}\right) \geqslant c$ a.e. We interpret $A$ as a multiplication operator in $\left(L^{2}(Q) \otimes L^{2}(\square)\right)^{n}$. For $\varepsilon>0$, we consider the following equation

$$
-\operatorname{div}_{x} \mathcal{T}_{-\varepsilon} A \mathcal{T}_{\varepsilon} \operatorname{grad}_{x} u_{\varepsilon}=f
$$

with $f \in L^{2}(Q) \otimes \mathbb{C}$. In this case, the term $\mathcal{T}_{-\varepsilon} \underset{\circ}{A} \mathcal{T}_{\varepsilon} \operatorname{grad}_{x} u_{\varepsilon}$ boils down to the familiar expression

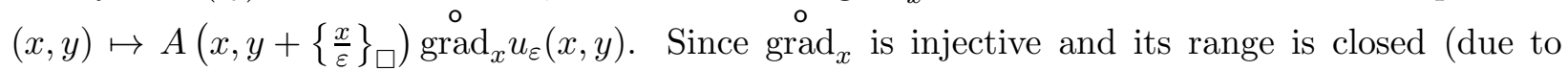
the Poincaré inequality), a direct application of Theorem 3.1 and the fact that $\mathcal{T}_{\varepsilon} u=u$ for $u \in$ $\operatorname{ker}\left(\operatorname{grad}_{y}^{\#}\right)$ imply the following:

Corollary 5.8. Let $A$ be given as above and $u_{\varepsilon}$ be the unique solution of (34). Then

$$
\mathcal{T}_{\varepsilon} u_{\varepsilon} \rightarrow u \text { strongly in } L^{2}(Q) \otimes L^{2}(\square), \quad \mathcal{T}_{\varepsilon} \operatorname{grad}_{x} u_{\varepsilon} \rightarrow \operatorname{grad}_{x} u+v \quad \text { weakly in }\left(L^{2}(Q) \otimes L^{2}(\square)\right)^{n},
$$

where $u \in \operatorname{dom}\left(\operatorname{grad}_{x}\right) \otimes \mathbb{C}$ and $v \in \overline{\operatorname{ran}}\left(\operatorname{grad}_{y}^{\#}\right)$ are the unique solution to

$$
\begin{aligned}
-\operatorname{div}_{x} P A\left(\operatorname{grad}_{x} u+v\right) & =f, \\
-\operatorname{div}_{y}^{\#} A\left(\operatorname{grad}_{x} u+v\right) & =0 .
\end{aligned}
$$

Above, $P$ is the projection to constant functions (in the $y$-variable), i.e., $P \varphi=\int_{\square} \varphi d y$.

Remark 5.9. In order to transform the above (two-scale) homogenized problem (35) into the usual one-scale form (see [1] for detailed investigation of such two-scale effective equations), we might introduce the following (uniquely defined) correctors $\varphi_{i} \in \operatorname{dom}\left(\operatorname{grad}_{y}^{\#}\right)(i \in\{1, \ldots, n\})$ by

$$
-\operatorname{div}_{y}^{\#} A\left(e_{i}+\operatorname{grad}_{y}^{\#} \varphi_{i}\right)=0, \quad \int_{\square} \varphi_{i}=0 .
$$

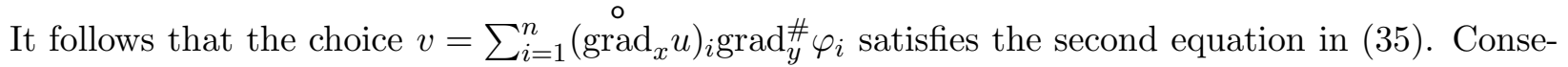
quently, $u$ is the solution of the equation

$$
-\operatorname{div}_{x} A_{\text {hom }^{\operatorname{grad}}} \stackrel{\circ}{x}=f,
$$

where $A_{\text {hom }} e_{i} \cdot e_{j}=\int_{\square} A\left(e_{i}+\operatorname{grad}_{y}^{\#} \varphi_{i}\right) \cdot\left(e_{j}+\operatorname{grad}_{y}^{\#} \varphi_{j}\right) d y$. The last equation is the classical form of the homogenized equation. Notice, however, that the result presented in Corollary 5.8 is slightly different from the classical result; as the notion of convergence is different. We also refer to [4, Remark on page 32] for a similar statement in the stochastic setting. 


\subsection{Stochastic homogenization of Maxwell's equations}

In this section we consider stochastic homogenization of Maxwell's equations. We refer to [50, 17] for the treatment of Maxwell's equations in the periodic setting using two-scale convergence arguments. The stochastic-periodic case is treated in [33] that is based on the notion of stochastic two-scale convergence from [4]. However, our approach is different, it relies on the operator theoretic formulation and on the unfolding strategy, in fact, the homogenization result Corollary 5.10 readily follows from the abstract Theorem 4.10. Also, we treat a more general situation-in contrast to our case - in [33] the assumptions on the coefficients do not allow for jumps or for regions, where the conductivity or dielectricity vanish. Moreover, as far as we know, the corrector type result Corollary 5.16 is not presented earlier in the stochastic setting, see [50, Theorem 3.3] for a similar periodic corrector result.

Let $Q \subseteq \mathbb{R}^{3}$ be open and $(\Omega, \Sigma, \mu, \tau)$ be a probability space satisfying Assumption 1 (with $n=3$ ). In this section we consider the stochastic unfolding operator $\mathcal{T}_{\varepsilon}: L^{2}(Q) \otimes L^{2}(\Omega) \rightarrow L^{2}(Q) \otimes L^{2}(\Omega)$ defined in (7). The role of $C_{d}$ and $C_{s}$ in this setting is played by the operators $\operatorname{curl}_{x}$ and $\operatorname{curl}_{\omega}$, respectively.

We set $H_{0}=H_{1}=\left(L^{2}(Q) \otimes L^{2}(\Omega)\right)^{3}$ and $H=H_{0} \oplus H_{1}$. We consider $\nu_{0}>0, \eta_{0}, \sigma_{0}, \mu_{0} \in$ $L^{\infty}(Q \times \Omega)^{3 \times 3}$ such that $\eta_{0}$ and $\mu_{0}$ are Hermitian a.e. and there exists $c>0$ such that

$$
\nu \eta_{0}+\operatorname{Re}\left(\sigma_{0}\right) \geqslant c \quad\left(\text { for all } \nu>\nu_{0}\right), \quad \mu_{0} \geqslant c .
$$

For $\varepsilon>0$ and $(f, g) \in L_{\nu}^{2}\left(\mathbb{R} ; L^{2}(Q)^{3} \oplus L^{2}(Q)^{3}\right)$, we consider the following system of equations

$$
\bar{B}_{\varepsilon}\left(\begin{array}{l}
u_{\varepsilon} \\
q_{\varepsilon}
\end{array}\right)=\left(\begin{array}{l}
f \\
g
\end{array}\right) \text {, where } B_{\varepsilon}=\mathcal{T}_{-\varepsilon}\left(\begin{array}{cc}
\partial_{t, \nu} \eta_{0}+\sigma_{0} & 0 \\
0 & \partial_{t, \nu} \mu_{0}
\end{array}\right) \mathcal{T}_{\varepsilon}+\left(\begin{array}{cc}
0 & -\operatorname{curl}_{x} \\
0 & 0 \\
\operatorname{curl}_{x} & 0
\end{array}\right) .
$$

The above system represents a system of Maxwell's equations with random and oscillating coefficients (the oscillating coefficients have the form $\mathcal{T}_{-\varepsilon} \eta_{0} \mathcal{T}_{\varepsilon}$ and similarly for $\sigma_{0}$ and $\mu_{0}$ ). According to Theorem 4.1 there exists a unique solution of the above equation $\left(u_{\varepsilon}, q_{\varepsilon}\right) \in L_{\nu}^{2}(\mathbb{R} ; H)$. Moreover, a direct application of Theorem 4.10 yields the following:

Corollary 5.10. Let $\eta_{0}, \sigma_{0}, \mu_{0}$ be given as above and let $\left(u_{\varepsilon}, q_{\varepsilon}\right) \in L_{\nu}^{2}(\mathbb{R} ; H)$ be the unique solution to (37). We have

$$
\mathcal{T}_{\varepsilon}\left(u_{\varepsilon}, q_{\varepsilon}\right) \rightarrow\left(\iota_{s} u, \iota_{s} q\right) \quad \text { weakly in } L_{\nu}^{2}(\mathbb{R}, H) .
$$

Above, $\iota_{s}$ denotes the canonical embedding $\iota_{s}: \operatorname{ker}\left(\operatorname{curl}_{\omega}\right) \hookrightarrow H_{0}=H_{1}$ and

$$
(u, q) \in L_{\nu}^{2}\left(\mathbb{R} ; \operatorname{ker}\left(\operatorname{curl}_{\omega}\right) \oplus \operatorname{ker}\left(\operatorname{curl}_{\omega}\right)\right)
$$

denotes the unique solution to

$\bar{B}_{\text {hom }}\left(\begin{array}{c}u \\ q\end{array}\right)=\left(\begin{array}{c}\iota_{s}^{*} f \\ \iota_{s}^{*} g\end{array}\right)$, where $B_{\text {hom }}=\left(\begin{array}{cc}\partial_{t, \nu} \iota_{s}^{*} \eta_{0} \iota_{s}+\iota_{s}^{*} \sigma_{0} \iota_{s} & 0 \\ 0 & \partial_{t, \nu} \iota_{s}^{*} \mu_{0} \iota_{s}\end{array}\right)+\left(\begin{array}{cc}0 & -\overline{\iota_{s}^{*} \operatorname{curl}_{x} \iota_{s}} \\ \iota_{s}^{*} \operatorname{curl}_{x} \iota_{s} & 0\end{array}\right)$.

Note that the fact $(u, q) \in L_{\nu}^{2}\left(\mathbb{R} ; \operatorname{ker}\left(\operatorname{curl}_{\omega}\right) \oplus \operatorname{ker}\left(\operatorname{curl}_{\omega}\right)\right)$ does not imply in general that the limit solution is deterministic (or shift-invariant), i.e., the solution still depends on the probability space variable $\omega$. In the following we present an equivalent formulation of the above equation 
by decomposing the solution $(u, q)$ to its deterministic (shift-invariant) and random parts. In particular, the deterministic part satisfies an effective Maxwell system and the random part is given by a suitable corrector equation. We introduce the following canonical embeddings

$$
\iota_{0}: L_{\mathrm{inv}}^{2}(\Omega)^{3} \hookrightarrow \operatorname{ker}\left(\operatorname{curl}_{\omega}\right), \quad \iota_{1}: L_{\mathrm{pot}}^{2}(\Omega) \hookrightarrow \operatorname{ker}\left(\operatorname{curl}_{\omega}\right) .
$$

Moreover, we consider the transformation

$$
T:=\left(\begin{array}{cccc}
\iota_{0} & \iota_{1} & 0 & 0 \\
0 & 0 & \iota_{0} & \iota_{1}
\end{array}\right): L_{\mathrm{inv}}^{2}(\Omega)^{3} \oplus L_{\mathrm{pot}}^{2}(\Omega) \oplus L_{\mathrm{inv}}^{2}(\Omega)^{3} \oplus L_{\mathrm{pot}}^{2}(\Omega) \rightarrow \operatorname{ker}\left(\operatorname{curl}_{\omega}\right) \oplus \operatorname{ker}\left(\operatorname{curl}_{\omega}\right)
$$

that is unitary (by Lemma 5.6). As a result of this, we obtain the following:

Corollary 5.11. Let $(u, q) \in L_{\nu}^{2}\left(\mathbb{R} ; \operatorname{ker}\left(\operatorname{curl}_{\omega}\right) \oplus \operatorname{ker}\left(\operatorname{curl}_{\omega}\right)\right)$ be the solution of (38). Then

$$
\left(\begin{array}{c}
u_{0} \\
\chi_{1} \\
q_{0} \\
\chi_{2}
\end{array}\right):=T^{-1}(u, q) \in L_{\nu}^{2}\left(\mathbb{R} ;\left(\left(L^{2}(Q) \otimes L_{\mathrm{inv}}^{2}(\Omega)\right)^{3} \oplus\left(L^{2}(Q) \otimes L_{\mathrm{pot}}^{2}(\Omega)\right)\right)^{2}\right)
$$

is the unique solution to

$$
\overline{T^{-1} B_{\mathrm{hom}} T}\left(\begin{array}{c}
u_{0} \\
\chi_{1} \\
q_{0} \\
\chi_{2}
\end{array}\right)=T^{-1}\left(\begin{array}{c}
\iota_{s}^{*} f \\
\iota_{s}^{*} g
\end{array}\right)
$$

Remark 5.12. Dropping the notation for the embeddings $\iota_{s}, T$ and closure bars, system (39) reads

$$
\begin{aligned}
\partial_{t, \nu} P_{\text {inv }} \eta_{0}\left(u_{0}+\chi_{1}\right)+P_{\text {inv }} \sigma_{0}\left(u_{0}+\chi_{1}\right)-\operatorname{curl}_{x} q_{0} & =f \\
\partial_{t, \nu} P_{\text {pot }} \eta_{0}\left(u_{0}+\chi_{1}\right)+P_{\text {pot }} \sigma_{0}\left(u_{0}+\chi_{1}\right) & =0 \\
\circ & \\
\partial_{t, \nu} P_{\text {inv }} \mu_{0}\left(q_{0}+\chi_{2}\right)+\operatorname{curl}_{x} u_{0} & =g \\
\partial_{t, \nu} P_{\text {pot }} \mu_{0}\left(q_{0}+\chi_{2}\right) & =0 .
\end{aligned}
$$

Note that in the second equation we used that $P_{\text {pot }} \operatorname{curl}_{x}\left(q_{0}+\chi_{2}\right)=0$ since $P_{\text {pot }} q_{0}=0$ and $P_{\text {pot }} \operatorname{curl}_{x} \chi_{2}=0$ (that can be obtained by a direct computation) and in the fourth equation we use $P_{\text {pot }} \operatorname{curl}_{x}\left(u_{0}+\chi_{1}\right)=0$ (obtained similarly as the previous claim). We regard the first and third equations as the effective Maxwell system for the (averaged) variable $\left(u_{0}, q_{0}\right)$ and the second and fourth equations are corrector equations which allow us to express $\left(\chi_{1}, \chi_{2}\right)$ as functions of $\left(u_{0}, q_{0}\right)$. In particular, the second and fourth equations imply that (additionally assuming that $\eta_{0}$ is positive-definite)

$$
\begin{aligned}
& \chi_{1}(t)=-\chi_{\eta_{0}}^{i} u_{0}^{i}(t)+e^{-A t}\left(\chi_{\eta_{0}}^{i} u_{0}^{i}(0)+\chi_{1}(0)\right)+\int_{0}^{t} e^{-A(t-\tau)} A\left(\chi_{\eta_{0}}^{i}-\chi_{\sigma_{0}}^{i}\right) u_{0}^{i}(\tau) d \tau, \\
& \chi_{2}(t)=-\chi_{\mu_{0}}^{i} q_{0}^{i}(t)+\chi_{\mu_{0}}^{i} q_{0}^{i}(0)+\chi_{2}(0),
\end{aligned}
$$

where $A=\eta_{0}^{-1} \sigma_{0}$ and $\chi_{\eta_{0}}^{i}, \chi_{\sigma_{0}}^{i}, \chi_{\mu_{0}}^{i} \in L^{2}(Q) \otimes L_{\text {pot }}^{2}(\Omega)(i \in\{1,2,3\})$ are the unique solutions to (respectively)

$$
P_{\mathrm{pot}} \eta_{0}\left(e_{i}-\chi_{\eta_{0}}^{i}\right)=0, \quad P_{\mathrm{pot}} \sigma_{0}\left(e_{i}-\chi_{\sigma_{0}}^{i}\right)=0, \quad P_{\mathrm{pot}} \mu_{0}\left(e_{i}-\chi_{\mu_{0}}^{i}\right)=0 .
$$


The derivation of these formulas is analogous to the periodic case [50] (see also [33] for the periodicstochastic case). In this sense, the effective equations for the averaged variables $\left(u_{0}, q_{0}\right)$ are non-local in time. This so-called memory effect has also been observed in [47] and [49] for coefficients that are not necessarily periodic or stochastic.

Remark 5.13 (Corrector equations). The equations in (40) are standard corrector equations in stochastic homogenization and might be brought into the form of an elliptic partial differential equation on $\mathbb{R}^{3}$. For simplicity let us consider the first equation in (40) and assume that $\eta_{0}$ does not depend on the physical space variable, i.e., $\eta_{0}(x, \omega)=\eta_{0}(\omega)$. The equation is equivalent to

$$
\int_{\Omega} \eta_{0}(\omega)\left(e_{i}-\chi_{\eta_{0}}^{i}(\omega)\right) \cdot \chi(\omega) d \mu(\omega)=0 \quad \text { for all } \chi \in L_{\text {pot }}^{2}(\Omega)
$$

which is a variational problem in the $L^{2}$-probability space. It turns out that for $\mu$-almost every $\omega \in \Omega$, the vector field $x \mapsto \chi_{\eta_{0}}^{i}\left(\tau_{x} \omega\right)$ has a potential $\varphi(\omega, \cdot) \in H_{\text {loc }}^{1}\left(\mathbb{R}^{3}\right)$ that is a distributional solution to

$$
-\nabla \cdot\left(\eta_{0}\left(\tau_{x} \omega\right)\left(e_{i}-\nabla \varphi(\omega, x)\right)\right)=0 \quad \text { in } \mathbb{R}^{3} .
$$

On the other hand, by standard theory in stochastic homogenization there exists a unique random field $\varphi: \Omega \times \mathbb{R}^{3} \rightarrow \mathbb{R}$ that is a distributional solution to (42) for $\mu$-a.e. $\omega \in \Omega$ such that $\nabla \varphi$ is stationary (i.e., $\nabla \varphi(\omega, x+z)=\nabla \varphi\left(\tau_{z} \omega, x\right)$ for $\mu$-a.e. $\omega \in \Omega$ and a.e. $\left.x, z \in \mathbb{R}^{3}\right)$, square integrable $\int_{\Omega}|\nabla \varphi(\omega, x)|^{2} d \mu(\omega)<\infty$, mean-free $\int_{\Omega} \nabla \varphi d \mu=0$, and anchored in the sense of $\int_{(0,1)^{d}} \varphi(\omega, x) d x=$ $0, \mu$-a.s. With this solution we recover the random vector field $\chi_{\eta_{0}}^{i}(\omega):=\nabla \varphi(\omega, 0)$ solving (41), see $[22$, Section 2.2] for details.

Note that both formulations, (41) and (42), are not accessible to a direct numerical approximation, since in the case of (41) a typical example for the probability measure $\mu$ would be a product-measure of the form $\hat{\mu}^{\otimes \mathbb{R}^{3}}$, while (42) is posed on the unbounded domain $\mathbb{R}^{3}$. A standard approximation scheme is the so-called periodization method, where in (42) the domain $\mathbb{R}^{3}$ is replaced by a large torus, say $L \square$ with $L \gg 1$, see, e.g., [5].

Corrector type results for Maxwell equations. First, we recall a (standard) mollification procedure from [30] (see also [45, 31]). For $\delta>0$, we consider the bounded operator $\mathcal{F}_{\delta}:=\left(1+\delta \partial_{t, \nu}\right)^{-1}$. We remark that $\mathcal{F}_{\delta} \rightarrow 1$ as $\delta \rightarrow 0$ in the strong operator topology and that for any $\varphi \in \operatorname{dom}\left(\bar{B}_{\varepsilon}\right)$, we have $\mathcal{F}_{\delta} \varphi \in \operatorname{dom}\left(B_{\varepsilon}\right)$ and $\mathcal{F}_{\delta} \bar{B}_{\varepsilon} \varphi=B_{\varepsilon} \mathcal{F}_{\delta} \varphi$ and the same statements hold if we replace $B_{\varepsilon}$ by $B_{\text {hom }}$. Using $\mathcal{F}_{\delta}$ and the properties of $\left(u_{\varepsilon}, q_{\varepsilon}\right)$, we obtain the following corrector type results. Before that, we provide another auxiliary result.

Lemma 5.14. We have

$$
\overline{\iota_{s}^{*} \operatorname{curl}_{x} \iota_{s}}=\iota_{s}^{*} \operatorname{curl}_{x} \iota_{s} \text { and } \overline{\iota_{s}^{*} \operatorname{curl}_{x} \iota_{s}}=\iota_{s}^{*} \operatorname{curl}_{x} \iota_{s} .
$$

Proof. Since $\operatorname{curl}_{x}$ and $\operatorname{curl}_{0}$ are closed linear operators, the claim follows, if we show that the application of $\operatorname{curl}_{x}$ and $\operatorname{curl}_{x}$ leaves $\operatorname{ker}\left(\operatorname{curl}_{\omega}\right)$ invariant. This, however, follows from the fact that $\operatorname{curl}_{x}$ and $\operatorname{curl}_{\omega}\left(\operatorname{curl}_{x}\right.$ and $\left.\operatorname{curl}_{\omega}\right)$ commute on the intersection of their domains.

In order to avoid clutter in notation, in the following we disregard the notation for the embedding $\iota_{s}$ and $T$. 
Proposition 5.15. Let $\delta>0,\left(u_{\varepsilon}, q_{\varepsilon}\right)$ be the solution of (37) and $\left(u_{0}, \chi_{1}, q_{0}, \chi_{2}\right)$ be the solution of (39). Then

$$
\left\|\mathcal{F}_{\delta}\left(u_{\varepsilon}-\mathcal{T}_{-\varepsilon} \chi_{1}-u_{0}\right)\right\|_{L_{\nu}^{2}\left(\mathbb{R} ; H_{0}\right)}+\left\|\mathcal{F}_{\delta}\left(q_{\varepsilon}-\mathcal{T}_{-\varepsilon} \chi_{2}-q_{0}\right)\right\|_{L_{\nu}^{2}\left(\mathbb{R} ; H_{1}\right)} \rightarrow 0 \quad \text { as } \varepsilon \rightarrow 0 .
$$

Proof. Using the facts that

$$
\operatorname{Re}\left\langle\left(\begin{array}{c}
u_{\varepsilon} \\
q_{\varepsilon}
\end{array}\right), \mathcal{T}_{-\varepsilon}\left(\begin{array}{cc}
\partial_{t, \nu} \eta_{0}+\sigma_{0} & 0 \\
0 & \partial_{t, \nu} \mu_{0}
\end{array}\right) \mathcal{T}_{\varepsilon}\left(\begin{array}{l}
u_{\varepsilon} \\
q_{\varepsilon}
\end{array}\right)\right\rangle \geqslant c\left\|\left(\begin{array}{l}
u_{\varepsilon} \\
q_{\varepsilon}
\end{array}\right)\right\|^{2}
$$

(using the assumptions on $\eta, \sigma, \mu$, see (36)) and that $\left(\begin{array}{cc}0 & -\operatorname{curl}_{x} \\ 0 & 0\end{array}\right)$ is skew-self-adjoint, we obtain

$$
\begin{aligned}
& \left\|\mathcal{F}_{\delta}\left(u_{\varepsilon}-\mathcal{T}_{-\varepsilon} \chi_{1}-u_{0}\right)\right\|_{L_{\nu}^{2}\left(\mathbb{R} ; H_{0}\right)}^{2}+\left\|\mathcal{F}_{\delta}\left(q_{\varepsilon}-\mathcal{T}_{-\varepsilon} \chi_{2}-q_{0}\right)\right\|_{L_{\nu}^{2}\left(\mathbb{R} ; H_{1}\right)}^{2} \\
\leqslant & \frac{1}{c} \operatorname{Re}\left\langle B_{\varepsilon} \mathcal{F}_{\delta}\left(\begin{array}{c}
u_{\varepsilon}-\mathcal{T}_{-\varepsilon} \chi_{1}-u_{0} \\
q_{\varepsilon}-\mathcal{T}_{-\varepsilon} \chi_{2}-q_{0}
\end{array}\right), \mathcal{F}_{\delta}\left(\begin{array}{c}
u_{\varepsilon}-\mathcal{T}_{-\varepsilon} \chi_{1}-u_{0} \\
q_{\varepsilon}-\mathcal{T}_{-\varepsilon} \chi_{2}-q_{0}
\end{array}\right)\right\rangle \\
= & \frac{1}{c} \operatorname{Re}\left\langle\mathcal{F}_{\delta} \bar{B}_{\varepsilon}\left(\begin{array}{c}
u_{\varepsilon} \\
q_{\varepsilon}
\end{array}\right), \mathcal{F}_{\delta}\left(\begin{array}{c}
u_{\varepsilon}-\mathcal{T}_{-\varepsilon} \chi_{1}-u_{0} \\
q_{\varepsilon}-\mathcal{T}_{-\varepsilon} \chi_{2}-q_{0}
\end{array}\right)\right\rangle \\
& -\frac{1}{c} \operatorname{Re}\left\langle B_{\varepsilon} \mathcal{F}_{\delta}\left(\begin{array}{c}
u_{0}+\mathcal{T}_{-\varepsilon} \chi_{1} \\
q_{0}+\mathcal{T}_{-\varepsilon} \chi_{2}
\end{array}\right), \mathcal{F}_{\delta}\left(\begin{array}{c}
u_{\varepsilon}-\mathcal{T}_{-\varepsilon} \chi_{1}-u_{0} \\
q_{\varepsilon}-\mathcal{T}_{-\varepsilon} \chi_{2}-q_{0}
\end{array}\right)\right\rangle .
\end{aligned}
$$

Above, we use that $\mathcal{F}_{\delta}\left(\begin{array}{c}\mathcal{T}_{-\varepsilon} \chi_{1}+u_{0} \\ \mathcal{T}_{-\varepsilon} \chi_{2}+q_{0}\end{array}\right) \in \operatorname{dom}\left(B_{\varepsilon}\right)$. This can be seen using the facts that

$$
\mathcal{F}_{\delta}\left(\begin{array}{l}
u_{0}+\chi_{1} \\
q_{0}+\chi_{2}
\end{array}\right) \in \operatorname{dom}\left(B_{\text {hom }}\right)
$$

and that $\mathcal{T}_{-\varepsilon}$ and $\mathcal{F}_{\delta}$ commute and, thus,

$$
\mathcal{F}_{\delta}\left(\begin{array}{l}
\mathcal{T}_{-\varepsilon} \chi_{1}+u_{0} \\
\mathcal{T}_{-\varepsilon} \chi_{2}+q_{0}
\end{array}\right) \in \operatorname{dom}\left(\left(\begin{array}{cc}
0 & -\operatorname{curl}_{x} \\
\circ & 0
\end{array}\right)\right)
$$

by Lemma 5.14. Since $\left(u_{\varepsilon}, q_{\varepsilon}\right)$ solves problem $(37)$ and $(f, g) \in \operatorname{ker}\left(\mathcal{T}_{\varepsilon}-1\right)$, it follows that the first term on the right-hand side satisfies

$$
\begin{aligned}
\frac{1}{c} \operatorname{Re}\left\langle\mathcal{F}_{\delta} \bar{B}_{\varepsilon}\left(\begin{array}{c}
u_{\varepsilon} \\
q_{\varepsilon}
\end{array}\right), \mathcal{F}_{\delta}\left(\begin{array}{c}
u_{\varepsilon}-\mathcal{T}_{-\varepsilon} \chi_{1}-u_{0} \\
q_{\varepsilon}-\mathcal{T}_{-\varepsilon} \chi_{2}-q_{0}
\end{array}\right)\right\rangle & =\frac{1}{c} \operatorname{Re}\left\langle\mathcal{F}_{\delta}\left(\begin{array}{c}
f \\
g
\end{array}\right), \mathcal{F}_{\delta}\left(\begin{array}{c}
u_{\varepsilon}-\mathcal{T}_{-\varepsilon} \chi_{1}-u_{0} \\
q_{\varepsilon}-\mathcal{T}_{-\varepsilon} \chi_{2}-q_{0}
\end{array}\right)\right\rangle \\
& =\frac{1}{c} \operatorname{Re}\left\langle\mathcal{F}_{\delta}\left(\begin{array}{c}
f \\
g
\end{array}\right), \mathcal{F}_{\delta}\left(\begin{array}{c}
\mathcal{T}_{\varepsilon} u_{\varepsilon}-\chi_{1}-u_{0} \\
\mathcal{T}_{\varepsilon} q_{\varepsilon}-\chi_{2}-q_{0}
\end{array}\right)\right\rangle .
\end{aligned}
$$

The last expression vanishes in the limit $\varepsilon \rightarrow 0$ since $\left(u_{\varepsilon}, q_{\varepsilon}\right) \stackrel{2}{\rightarrow}\left(u_{0}+\chi_{1}, q_{0}+\chi_{2}\right)$. We treat the 
second term on the right-hand side of (43) as follows:

$$
\begin{aligned}
& \operatorname{Re}\left\langle B_{\varepsilon} \mathcal{F}_{\delta}\left(\begin{array}{c}
u_{0}+\mathcal{T}_{-\varepsilon} \chi_{1} \\
q_{0}+\mathcal{T}_{-\varepsilon} \chi_{2}
\end{array}\right), \mathcal{F}_{\delta}\left(\begin{array}{c}
u_{\varepsilon}-\mathcal{T}_{-\varepsilon} \chi_{1}-u_{0} \\
q_{\varepsilon}-\mathcal{T}_{-\varepsilon} \chi_{2}-q_{0}
\end{array}\right)\right\rangle \\
& =\operatorname{Re}\left\langle\left(\left(\begin{array}{cc}
\partial_{t, \nu} \eta+\sigma & 0 \\
0 & \partial_{t, \nu} \mu
\end{array}\right)+\mathcal{T}_{\varepsilon}\left(\begin{array}{cc}
0 & -\operatorname{curl}_{x} \\
0 & 0 \\
\operatorname{curl} x_{x} & 0
\end{array}\right) \mathcal{T}_{-\varepsilon}\right) \mathcal{F}_{\delta}\left(\begin{array}{l}
u_{0}+\chi_{1} \\
q_{0}+\chi_{2}
\end{array}\right), \mathcal{F}_{\delta}\left(\begin{array}{l}
\mathcal{T}_{\varepsilon} u_{\varepsilon}-\chi_{1}-u_{0} \\
\mathcal{T}_{\varepsilon} q_{\varepsilon}-\chi_{2}-q_{0}
\end{array}\right)\right\rangle \\
& =\operatorname{Re}\left\langle\left(\left(\begin{array}{cc}
\partial_{t, \nu} \eta+\sigma & 0 \\
0 & \partial_{t, \nu} \mu
\end{array}\right)+\left(\begin{array}{cc}
0 & -\operatorname{curl}_{x} \\
0 & 0
\end{array}\right)\right) \mathcal{F}_{\delta}\left(\begin{array}{l}
u_{0}+\chi_{1} \\
q_{0}+\chi_{2}
\end{array}\right), \mathcal{F}_{\delta}\left(\begin{array}{l}
\mathcal{T}_{\varepsilon} u_{\varepsilon}-\chi_{1}-u_{0} \\
\mathcal{T}_{\varepsilon} q_{\varepsilon}-\chi_{2}-q_{0}
\end{array}\right)\right\rangle .
\end{aligned}
$$

In order to justify the second equality above, we compute

$$
\left(\begin{array}{cc}
0 & -\operatorname{curl}_{x} \\
\circ & 0
\end{array}\right) \mathcal{T}_{-\varepsilon} \mathcal{F}_{\delta}\left(\begin{array}{l}
u_{0}+\chi_{1} \\
q_{0}+\chi_{2}
\end{array}\right)=\left(\begin{array}{c}
\operatorname{curl}_{x} \mathcal{T}_{-\varepsilon} \mathcal{F}_{\delta}\left(q_{0}+\chi_{2}\right) \\
\circ \\
\operatorname{curl}_{x} \mathcal{T}_{-\varepsilon} \mathcal{F}_{\delta}\left(u_{0}+\chi_{1}\right)
\end{array}\right)=\left(\begin{array}{c}
\mathcal{T}_{-\varepsilon} \operatorname{curl}_{x} \mathcal{F}_{\delta}\left(q_{0}+\chi_{2}\right) \\
\mathcal{T}_{-\varepsilon} \operatorname{curl}_{x} \mathcal{F}_{\delta}\left(u_{0}+\chi_{1}\right)
\end{array}\right),
$$

where we used the commutation relations from Lemma $5.4(\mathrm{~b})$, the fact that $\mathcal{F}_{\delta}$ and $\operatorname{curl}_{\omega} \operatorname{commute}$ and that $\left(u_{0}+\chi_{1}\right) \in \operatorname{ker}\left(\operatorname{curl}_{\omega}\right)$ and $\left(q_{0}+\chi_{2}\right) \in \operatorname{ker}\left(\operatorname{curl}_{\omega}\right)$. Also, letting $\varepsilon \rightarrow 0$ in (44), the righthand side vanishes since $\left(u_{\varepsilon}, q_{\varepsilon}\right) \stackrel{2}{\rightarrow}\left(u_{0}+\chi_{1}, q_{0}+\chi_{2}\right)$. This concludes the proof.

If we, additionally, assume that $(f, g) \in \operatorname{dom}\left(\partial_{t, \nu}\right)$, it follows that $\left(u_{\varepsilon}, q_{\varepsilon}\right) \in \operatorname{dom}\left(\partial_{t, \nu}\right)$ and $\left(u_{\varepsilon}, q_{\varepsilon}\right) \in \operatorname{dom}\left(B_{\varepsilon}\right)$ (the analogous claims hold if we replace $B_{\varepsilon}$ by $B_{\text {hom }}$ and $\left(u_{\varepsilon}, q_{\varepsilon}\right)$ by $\left(u_{0}+\right.$ $\left.\left.\chi_{1}, q_{0}+\chi_{2}\right)\right)$. As a result of this, we obtain the following:

Corollary 5.16. Assume the same assumptions as in Corollary 5.10. Additionally, we assume that $(f, g) \in H_{\nu}^{1}(\mathbb{R} ; H)\left(=\operatorname{dom}\left(\partial_{t, \nu}\right)\right)$. Then

$$
\left\|u_{\varepsilon}-\mathcal{T}_{-\varepsilon} \chi_{1}-u_{0}\right\|_{L_{\nu}^{2}\left(\mathbb{R} ; H_{0}\right)}^{2}+\left\|q_{\varepsilon}-\mathcal{T}_{-\varepsilon} \chi_{2}-q_{0}\right\|_{L_{\nu}^{2}\left(\mathbb{R} ; H_{1}\right)}^{2} \rightarrow 0 \quad \text { as } \varepsilon \rightarrow 0 .
$$

Proof. Since $(f, g) \in H_{\nu}^{1}(\mathbb{R} ; H)$, we may apply the operator $\left(1+\partial_{t, \nu}\right)$ to both sides of the equations (37) and (38) to obtain

$$
\begin{aligned}
\bar{B}_{\varepsilon}\left(1+\partial_{t, \nu}\right)\left(\begin{array}{l}
u_{\varepsilon} \\
q_{\varepsilon}
\end{array}\right) & =\left(1+\partial_{t, \nu}\right)\left(\begin{array}{l}
f \\
g
\end{array}\right), \\
\bar{B}_{\mathrm{hom}}\left(1+\partial_{t, \nu}\right)\left(\begin{array}{l}
u_{0}+\chi_{1} \\
q_{0}+\chi_{2}
\end{array}\right) & =\left(1+\partial_{t, \nu}\right)\left(\begin{array}{l}
f \\
g
\end{array}\right) .
\end{aligned}
$$

We have that $w_{\varepsilon}:=\left(1+\partial_{t, \nu}\right)\left(\begin{array}{l}u_{\varepsilon} \\ q_{\varepsilon}\end{array}\right)$ and $w_{0}:=\left(1+\partial_{t, \nu}\right)\left(\begin{array}{l}u_{0}+\chi_{1} \\ q_{0}+\chi_{2}\end{array}\right)$ solve equations (37) and (38) with right-hand side $\left(1+\partial_{t, \nu}\right)\left(\begin{array}{l}f \\ g\end{array}\right)$ (instead of $\left(\begin{array}{l}f \\ g\end{array}\right)$ ). As a result of this, Proposition 5.15 implies that for any $\delta>0$, we have

$$
\left\|\mathcal{F}_{\delta}\left(w_{\varepsilon}-\mathcal{T}_{-\varepsilon} w_{0}\right)\right\|_{L_{\nu}^{2}(\mathbb{R} ; H)}^{2} \rightarrow 0 \quad \text { as } \varepsilon \rightarrow 0 .
$$

Setting $\delta=1$, the claim follows. 


\subsection{Stochastic homogenization of some mixed type equations}

A standard reference for homogenization of the wave and heat equation is [6], where general equations are treated in the framework of $\mathrm{H}$-convergence. In contrast to standard results for the wave or heat equation, the equation we consider features coefficients $\eta$ and $\sigma$, cf. (47), that are allowed to be alternatingly vanishing in some regions of the considered physical domain. This means that our setting contains equations of mixed alternating hyperbolic-parabolic type.

Let $Q \subseteq \mathbb{R}^{n}$ be open and $(\Omega, \Sigma, \mu, \tau)$ be a probability space satisfying Assumption 1 . We consider the stochastic unfolding operator $\mathcal{T}_{\varepsilon}: L^{2}(Q) \otimes L^{2}(\Omega) \rightarrow L^{2}(Q) \otimes L^{2}(\Omega)$ defined in (7). The role of $C_{d}$ and $C_{s}$ in this setting is played by the operators $\operatorname{grad}_{x}$ and $\operatorname{grad}_{\omega}$ (respectively).

Let $\nu_{0}>0, A \in L^{\infty}(Q \times \Omega)^{n \times n}$ be such that there exists $c>0$ with $A$ Hermitian a.e and $c \leqslant A(x, \omega) \leqslant \frac{1}{c}$ a.e.. Also, let $\eta, \sigma \in L^{\infty}(Q \times \Omega)$ be such that there exists $c>0$ with

$$
\operatorname{Re}(z \eta+\sigma) \geqslant c \quad\left(\text { for all } z \in \mathbb{C}_{\operatorname{Re} \geqslant \nu_{0}}\right) .
$$

Let $H_{0}=L^{2}(Q) \otimes L^{2}(\Omega), H_{1}=L^{2}(Q) \otimes L^{2}(\Omega)^{n}$ and $H=H_{0} \oplus H_{1}$. For $\varepsilon>0$ and $f \in L^{2}(Q)$, we consider the following system

$$
\bar{B}_{\varepsilon}\left(\begin{array}{l}
u_{\varepsilon} \\
q_{\varepsilon}
\end{array}\right)=\left(\begin{array}{l}
f \\
0
\end{array}\right), \quad \text { where } B_{\varepsilon}=\mathcal{T}_{-\varepsilon}\left(\begin{array}{cc}
\partial_{t, \nu} \eta+\sigma & 0 \\
0 & \partial_{t, \nu} A^{-1}
\end{array}\right) \mathcal{T}_{\varepsilon}+\left(\begin{array}{cc}
0 & \operatorname{div}_{x} \\
\circ & 0 \\
\operatorname{grad}_{x} & 0
\end{array}\right) .
$$

According to Theorem 4.1 the above system has a unique solution $\left(u_{\varepsilon}, q_{\varepsilon}\right) \in L_{\nu}^{2}(\mathbb{R} ; H)$.

Remark 5.17. Setting $w_{\varepsilon}=\partial_{t, \nu}^{-1} u_{\varepsilon}$, it follows that $w_{\varepsilon}$ solves the following wave equation

$$
\eta_{\varepsilon} \partial_{t, \nu}^{2} w_{\varepsilon}+\sigma_{\varepsilon} \partial_{t, \nu} w_{\varepsilon}-\operatorname{div}_{x} A_{\varepsilon} \operatorname{grad}_{x} w_{\varepsilon}=f,
$$

where the oscillating coefficients are given as the composition $A_{\varepsilon}:=\mathcal{T}_{-\varepsilon} A \mathcal{T}_{\varepsilon}, \eta_{\varepsilon}:=\mathcal{T}_{-\varepsilon} \eta \mathcal{T}_{\varepsilon}$ and $\sigma_{\varepsilon}:=\mathcal{T}_{-\varepsilon} \sigma \mathcal{T}_{\varepsilon}$.

Using our abstract homogenization result Theorem 4.10 we obtain:

Corollary 5.18. Let $A, \eta, \sigma$ be given as above and let $\left(u_{\varepsilon}, q_{\varepsilon}\right) \in L_{\nu}^{2}(\mathbb{R} ; H)$ be the unique solution to (46). Then

$$
\mathcal{T}_{\varepsilon}\left(u_{\varepsilon}, q_{\varepsilon}\right) \rightarrow\left(\iota_{s} u, \iota_{s^{*}} q\right) \quad \text { weakly in } L_{\nu}^{2}(\mathbb{R} ; H) .
$$

Above, $\iota_{s}$ and $\iota_{s^{*}}$ denote the canonical embeddings $\iota_{s}: \operatorname{ker}\left(\operatorname{grad}_{\omega}\right) \rightarrow H_{0}$ and $\iota_{s^{*}}: \operatorname{ker}\left(\operatorname{div}_{\omega}\right) \rightarrow H_{1}$, and $(u, q) \in L_{\nu}^{2}\left(\mathbb{R} ; \operatorname{ker}\left(\operatorname{grad}_{\omega}\right) \oplus \operatorname{ker}\left(\operatorname{div}_{\omega}\right)\right)$ is the unique solution to

$$
\begin{aligned}
& \bar{B}_{\text {hom }}\left(\begin{array}{l}
u \\
q
\end{array}\right)=\left(\begin{array}{c}
\iota_{s}^{*} f \\
0
\end{array}\right), \\
& B_{\text {hom }}=\left(\begin{array}{ccc}
\partial_{t, \nu} \iota_{s}^{*} \eta \iota_{s}+\iota_{s}^{*} \sigma \iota_{s} & 0 \\
0 & \partial_{t, \nu} \iota_{s}^{*} A^{-1} \iota_{s^{*}}
\end{array}\right)+\left(\begin{array}{cc}
\frac{0}{\iota^{\circ}} & \overline{\iota_{s}^{*} \operatorname{div}_{x} \iota_{s^{*}}} \\
\iota_{s^{*}}^{*} \operatorname{grad}_{x} \iota_{s} & 0
\end{array}\right) .
\end{aligned}
$$

Remark 5.19. In order to recover the classical form of the homogenized wave equation, we set $w=\partial_{t, \nu}^{-1} u$ to obtain (with the usual abuse of notation)

$$
P_{\mathrm{inv}} \eta \partial_{t, \nu}^{2} w+P_{\mathrm{inv}} \sigma \partial_{t, \nu} w+\operatorname{div}_{x} P_{\mathrm{inv}} q=f
$$


where $q$ satisfies $\left.P_{\operatorname{ker}(\operatorname{div} \omega}\right)\left(\partial_{t, \nu} A^{-1} q+\stackrel{\circ}{\operatorname{grad}_{x} \partial_{t, \nu} w}\right)=0\left(\right.$ here $\left.\left.P_{\operatorname{ker}(\operatorname{div} \omega}\right)=\iota_{s^{*}}^{*}\right)$. Applying $\partial_{t, \nu}^{-1}$, it follows that $P_{\operatorname{ker}\left(\operatorname{div}_{\omega}\right)} A^{-1} q=-\operatorname{grad}_{x} w$. As a result of this and using (32), we have that $A^{-1} q=$ $-\operatorname{grad}_{x} w+\chi$ where $\chi \in L^{2}(Q) \otimes L_{\text {pot }}^{2}(\Omega)$ (note that $\chi$ is uniquely determined). Also, since $q \in$ $\operatorname{ker}\left(\operatorname{div}_{\omega}\right)$, we have $-\operatorname{div}_{\omega} A\left(\operatorname{grad}_{x} w-\chi\right)=0$. Collecting these facts, we obtain that $w$ satisfies

$$
P_{\mathrm{inv}} \eta \partial_{t, \nu}^{2} w+P_{\mathrm{inv}} \sigma \partial_{t, \nu} w-\operatorname{div}_{x} P_{\mathrm{inv}} A\left(\stackrel{\circ}{\left.\operatorname{grad}_{x} w-\chi\right)}=f\right.
$$

where $\chi$ solves the usual corrector equation $-\operatorname{div}_{\omega} A\left(\stackrel{\circ}{\operatorname{grad}_{x} w}-\chi\right)=0$.

The above remark suggests the following corrector type statement $\mathcal{T}_{\varepsilon} \operatorname{grad}_{x} w_{\varepsilon}+\chi \rightarrow \stackrel{\circ}{\operatorname{grad}_{x} w}$ that is equivalent to $A^{-1} \mathcal{T}_{\varepsilon} q_{\varepsilon}-\chi \rightarrow P_{\operatorname{ker}\left(\operatorname{div}_{\omega}\right)} A^{-1} q=A^{-1} q-\chi$. We formalize this in the following.

Proposition 5.20 (Corrector type result). Let $f \in H_{\nu}^{1}\left(\mathbb{R} ; H_{0}\right)$, then

$$
\left\|\partial_{t, \nu}^{-1} u_{\varepsilon}-\partial_{t, \nu}^{-1} u\right\|_{L_{\nu}^{2}\left(\mathbb{R}, H_{0}\right)}+\left\|u_{\varepsilon}-u\right\|_{L_{\nu}^{2}\left(\mathbb{R} ; H_{0}\right)}+\left\|\mathcal{T}_{\varepsilon} q_{\varepsilon}-q\right\|_{L_{\nu}^{2}\left(\mathbb{R} ; H_{1}\right)} \rightarrow 0 \quad \text { as } \varepsilon \rightarrow 0 .
$$

Proof. This proof follows similar lines to the proof of Proposition 5.15. Using the assumptions on $A, \sigma$, and $\eta$, we have

$$
\operatorname{Re}\left\langle\left(\begin{array}{c}
w_{\varepsilon} \\
q_{\varepsilon}
\end{array}\right), \mathcal{T}_{\varepsilon}\left(\begin{array}{cc}
\partial_{t, \nu} \eta+\sigma & 0 \\
0 & \partial_{t, \nu} A^{-1}
\end{array}\right) \mathcal{T}_{-\varepsilon}\left(\begin{array}{c}
w_{\varepsilon} \\
q_{\varepsilon}
\end{array}\right)\right\rangle \geqslant c\left\|\left(\begin{array}{c}
w_{\varepsilon} \\
q_{\varepsilon}
\end{array}\right)\right\|^{2}
$$

As a result of this and by the skew-self-adjointness of $\left(\begin{array}{cc}0 & \operatorname{div}_{x} \\ 0 & 0 \\ \operatorname{grad}_{x} & 0\end{array}\right)$, we obtain

$$
\begin{aligned}
& \left\|u_{\varepsilon}-\mathcal{T}_{-\varepsilon} u\right\|_{L_{\nu}^{2}\left(\mathbb{R} ; H_{0}\right)}^{2}+\left\|q_{\varepsilon}-\mathcal{T}_{-\varepsilon} q\right\|_{L_{\nu}^{2}\left(\mathbb{R} ; H_{1}\right)}^{2} \\
\leqslant & \frac{1}{c} \operatorname{Re}\left\langle B_{\varepsilon}\left(\begin{array}{c}
u_{\varepsilon}-\mathcal{T}_{-\varepsilon} u \\
q_{\varepsilon}-\mathcal{T}_{-\varepsilon} q
\end{array}\right),\left(\begin{array}{c}
u_{\varepsilon}-\mathcal{T}_{-\varepsilon} u \\
q_{\varepsilon}-\mathcal{T}_{-\varepsilon} q
\end{array}\right)\right\rangle \\
= & \frac{1}{c} \operatorname{Re}\left\langle\left(\begin{array}{c}
f \\
0
\end{array}\right),\left(\begin{array}{c}
u_{\varepsilon}-\mathcal{T}_{-\varepsilon} u \\
q_{\varepsilon}-\mathcal{T}_{-\varepsilon} q
\end{array}\right)\right\rangle-\frac{1}{c} \operatorname{Re}\left\langle B_{\varepsilon}\left(\begin{array}{c}
\mathcal{T}_{-\varepsilon} u \\
\mathcal{T}_{-\varepsilon} q
\end{array}\right),\left(\begin{array}{c}
u_{\varepsilon}-\mathcal{T}_{-\varepsilon} u \\
q_{\varepsilon}-\mathcal{T}_{-\varepsilon} q
\end{array}\right)\right\rangle,
\end{aligned}
$$

where in the second equality we use the equation (46). The first term on the right-hand side vanishes in the limit $\varepsilon \rightarrow 0$ using Corollary 5.18 and that $\mathcal{T}_{\varepsilon} f=f$. The second term is treated as follows. We have

$$
\begin{gathered}
\operatorname{Re}\left\langle B_{\varepsilon}\left(\begin{array}{c}
\mathcal{T}_{-\varepsilon} u \\
\mathcal{T}_{-\varepsilon} q
\end{array}\right),\left(\begin{array}{c}
u_{\varepsilon}-\mathcal{T}_{-\varepsilon} u \\
q_{\varepsilon}-\mathcal{T}_{-\varepsilon} q
\end{array}\right)\right\rangle \\
=\operatorname{Re}\left\langle\left(\left(\begin{array}{cc}
\partial_{t, \nu} \eta+\sigma & 0 \\
0 & \partial_{t, \nu} A^{-1}
\end{array}\right)+\mathcal{T}_{\varepsilon}\left(\begin{array}{cc}
0 & \operatorname{div}_{x} \\
0 & \mathcal{T}_{-\varepsilon} \\
\operatorname{grad}_{x} & 0
\end{array}\right)\left(\begin{array}{l}
u \\
q
\end{array}\right),\left(\begin{array}{l}
\mathcal{T}_{\varepsilon} u_{\varepsilon}-u \\
\mathcal{T}_{\varepsilon} q_{\varepsilon}-q
\end{array}\right)\right\rangle\right. \\
\text { Similarly as in (45), we obtain } \mathcal{T}_{\varepsilon}\left(\begin{array}{cc}
0 & \operatorname{div}_{x} \\
0 & \\
\operatorname{grad}_{x} & 0
\end{array}\right) \mathcal{T}_{-\varepsilon}\left(\begin{array}{l}
u \\
q
\end{array}\right)=\left(\begin{array}{cc}
0 & \operatorname{div}_{x} \\
0 & \\
\operatorname{grad}_{x} & 0
\end{array}\right)\left(\begin{array}{l}
u \\
q
\end{array}\right) \text { and therefore }
\end{gathered}
$$
the above expression vanishes in the limit $\varepsilon \rightarrow 0$. This concludes the proof. 
Above, we assumed that the right-hand side $f$ is smooth for convenience, if this is not the case, we could use a mollification procedure as in Proposition 5.15. We briefly summarize the implications of the above proposition for the sequence $w_{\varepsilon}$ :

$$
w_{\varepsilon} \rightarrow w, \quad \partial_{t, \nu} w_{\varepsilon} \rightarrow \partial_{t, \nu} w, \quad \stackrel{\circ}{\operatorname{grad}_{x} w_{\varepsilon}}+\mathcal{T}_{-\varepsilon} \chi \rightarrow \stackrel{\circ}{\operatorname{grad}_{x} w} \quad\left(\text { strongly in } L_{\nu}^{2}\left(\mathbb{R} ; L^{2}(Q) \otimes L^{2}(\Omega)\right)\right)
$$

Remark 5.21. It is well-known that the classical homogenized wave equation (48) is an unsatisfactory approximation for (47) on large time scales $t \gtrsim \varepsilon^{-2}$ (see [12]). This fact is not reflected in our result ((49) is given in a norm accounting for the whole time line $t \in \mathbb{R})$ since we use a norm which is weighted with an exponential weight $e^{-2 \nu t}(\nu>0)$ that diminishes the effects on large time scales.

\section{Acknowledgments}

SN and MV acknowledge funding by the Deutsche Forschungsgemeinschaft (DFG, German Research Foundation) project number 405009441, and in the context of TU Dresden's Institutional Strategy "The Synergetic University".

\section{References}

[1] G. Allaire. Homogenization and two-scale convergence. SIAM J. Math. Anal., 23(6):1482-1518, 1992.

[2] K. T. Andrews and S. Wright. Stochastic homogenization of elliptic boundary-value problems with $L^{p}$-data. Asymptotic Analysis, 17(3):165-184, 1998.

[3] A. Bensoussan, J.-L. Lions, and G. Papanicolaou. Asymptotic analysis for periodic structures, volume 5 of Studies in Mathematics and its Applications. North-Holland Publishing Co., Amsterdam-New York, 1978.

[4] A. Bourgeat, A. Mikelić, and S. Wright. Stochastic two-scale convergence in the mean and applications. J. Reine Angew. Math., 456:19-51, 1994.

[5] A. Bourgeat and A. Piatnitski. Approximations of effective coefficients in stochastic homogenization. In Annales de l'IHP Probabilités et statistiques, volume 40, pages 153-165, 2004.

[6] S. Brahim-Otsmane, G. A. Francfort, and F. Murat. Correctors for the homogenization of the wave and heat equations. Journal de mathématiques pures et appliquées, 71(3):197-231, 1992.

[7] H. Brezis. Functional analysis, Sobolev spaces and partial differential equations. Springer Science \& Business Media, 2010.

[8] D. Cioranescu, A. Damlamian, and G. Griso. Periodic unfolding and homogenization. Comptes Rendus Mathematique, 335(1):99-104, 2002.

[9] D. Cioranescu, A. Damlamian, and G. Griso. The periodic unfolding method in homogenization. SIAM Journal on Mathematical Analysis, 40(4):1585-1620, 2008. 
[10] D. Cioranescu and P. Donato. An introduction to homogenization, volume 17 of Oxford Lecture Series in Mathematics and its Applications. The Clarendon Press, Oxford University Press, New York, 1999.

[11] S. Cooper and M. Waurick. Fibre homogenisation. Journal of Functional Analysis, 276(11):3363-3405, 2019.

[12] T. Dohnal, A. Lamacz, and B. Schweizer. Bloch-wave homogenization on large time scales and dispersive effective wave equations. Multiscale Model. Simul., 12(2):488-513, 2014.

[13] M. Heida. An extension of the stochastic two-scale convergence method and application. Asymptotic Analysis, 72(1-2):1-30, 2011.

[14] M. Heida, S. Neukamm, and M. Varga. Stochastic homogenization of $\Lambda$-convex gradient flows. arXiv preprint arXiv:1905.02562, 2019.

[15] V. V. Jikov, S. M. Kozlov, and O. A. Oleinik. Homogenization of differential operators and integral functionals. Springer Science \& Business Media, 2012.

[16] A. Kalauch, R. Picard, S. Siegmund, S. Trostorff, and M. M. Waurick. A Hilbert Space Perspective on Ordinary Differential Equations with Memory Term. Journal of Dynamics and Differential Equations, 26(2):369-399, 2014.

[17] G. Kristensson and N. Wellander. Homogenization of the maxwell equations at fixed frequency. SIAM Journal on Applied Mathematics, 64(1):170-195, 2003.

[18] D. Lukkassen, G. Nguetseng, and P. Wall. Two-scale convergence. International Journal of Pure and Applied Mathematics, 2(1):35-86, 2002.

[19] D. McGhee, R. Picard, S. Trostorff, and M. Waurick. Partial Differential Equations, chapter 16, pages 527-550. Wiley, 2014.

[20] A. Mielke, T. Roubiček, and U. Stefanelli. $\Gamma$-limits and relaxations for rate-independent evolutionary problems. Calc. Var. Partial Differential Equations, 31(3):387-416, 2008.

[21] F. Murat and L. Tartar. H-convergence. In Topics in the mathematical modelling of composite materials, pages 21-43. Springer, 2018.

[22] S. Neukamm. An introduction to the qualitative and quantitative theory of homogenization. Interdisciplinary Information Sciences, 24(1):1-48, 2018.

[23] S. Neukamm and M. Varga. Stochastic unfolding and homogenization of spring network models. Multiscale Modeling \& Simulation, 16(2):857-899, 2018.

[24] G. Nguetseng. A general convergence result for a functional related to the theory of homogenization. SIAM J. Math. Anal., 20(3):608-623, 1989.

[25] G. C. Papanicolaou and S. S. Varadhan. Boundary value problems with rapidly oscillating random coefficients. Random fields, 1:835-873, 1979.

[26] R. Picard. A structural observation for linear material laws in classical mathematical physics. Mathematical Methods in the Applied Sciences, 32:1768-1803, 2009. 
[27] R. Picard and D. McGhee. Partial Differential Equations: A unified Hilbert Space Approach,, volume 55. Expositions in Mathematics. DeGruyter, Berlin, 2011.

[28] R. Picard, S. Trostorff, and M. Waurick. On Evolutionary Equations with Material Laws Containing Fractional Integrals. Math. Meth. Appl. Sci., 38(15):3141-3154, 2015.

[29] R. Picard, S. Trostorff, and M. Waurick. Well-posedness via monotonicity. an overview. In Operator Semigroups Meet Complex Analysis, Harmonic Analysis and Mathematical Physics. Operator Theory: Advances and Applications, volume 250, pages 397-452, 2015.

[30] R. Picard, S. Trostorff, and M. Waurick. On maximal regularity for a class of evolutionary equations. Journal of Mathematical Analysis and Applications, 449(2):1368-1381, 2017.

[31] R. Picard, S. Trostorff, M. Waurick, and M. Wehowski. On Non-Autonomous Evolutionary Problems. Journal of Evolution Equations, 13:751-776, 2013.

[32] A. Süß and M. Waurick. A Solution Theory for a General Class of SPDEs. Stochastics and Partial Differential Equations: Analysis and Computations, 5(2):278-318, 2017.

[33] J. F. Tachago and H. Nnang. Stochastic-periodic homogenization of maxwells equations with linear and periodic conductivity. Acta Mathematica Sinica, English Series, 33(1):117-152, 2017.

[34] A. ter Elst, G. Gorden, and M. Waurick. The Dirichlet-to-Neumann operator for divergence form problems. Annali di Matematica Pura ed Applicata, 198(1):177-203, 2019. doi.org/10.1007/s10231-018-0768-2.

[35] S. Trostorff. Exponential stability for linear evolutionary equations. Asymptot. Anal., 85(34):179-197, 2013.

[36] S. Trostorff. Exponential stability for second order evolutionary problems. J. Math. Anal. Appl., 429(2):1007-1032, 2015.

[37] S. Trostorff and M. Waurick. A note on elliptic type boundary value problems with maximal monotone relations. Mathematische Nachrichten, 287(13):1545-1558, 2014.

[38] M. Varga. Stochastic unfolding and homogenization of evolutionary gradient systems. PhD thesis, TU Dresden, in preparation.

[39] A. Visintin. Towards a two-scale calculus. ESAIM: Control, Optimisation and Calculus of Variations, 12(3):371-397, 2006.

[40] M. Waurick. Limiting Processes in Evolutionary Equations - A Hilbert Space Approach to Homogenization. Dissertation, Technische Universität Dresden, 2011. http://nbnresolving.de/urn:nbn:de:bsz:14-qucosa-67442.

[41] M. Waurick. A Hilbert Space Approach to Homogenization of Linear Ordinary Differential Equations Including Delay and Memory Terms. Mathematical Methods in the Applied Sciences, 35(9):1067-1077, 2012. 
[42] M. Waurick. Homogenization of a class of linear partial differential equations. Asymptotic Analysis, 82:271-294, 2013.

[43] M. Waurick. G-convergence of linear differential equations. Journal of Analysis and its Applications, 33(4):385-415, 2014.

[44] M. Waurick. Homogenization in fractional elasticity. SIAM J. Math. Anal., 46(2):1551-1576, 2014.

[45] M. Waurick. On non-autonomous integro-differential-algebraic evolutionary problems. Mathematical Methods in the Applied Sciences, 38(4):665-676, 2015.

[46] M. Waurick. On the continuous dependence on the coefficients of evolutionary equations. Habilitation, Technische Universität Dresden, 2016. http://arxiv.org/abs/1606.07731.

[47] M. Waurick. On the homogenization of partial integro-differential-algebraic equations. Operators and Matrices, 10(2):247-283, 2016.

[48] M. Waurick. Continuous dependence on the coefficients for a class of non-autonomous evolutionary equations. In Proceedings of the Special Semester 2016 in RICAM in Linz. Birkhäuser/Springer Basel AG, Basel, 2017. Acccepted.

[49] M. Waurick. Nonlocal H-convergence. Calculus of Variations and Partial Differential Equations, 57(6):46 pages, 2018.

[50] N. Wellander. Homogenization of the Maxwell equations. Case I. Linear theory. Appl. Math., 46(1):29-51, 2001.

[51] V. V. Zhikov and A. Pyatnitskii. Homogenization of random singular structures and random measures. Izvestiya: Mathematics, 70(1):19-67, 2006. 